\title{
TGF- $\beta$ modulates cell fate in human ES cell-derived foregut endoderm by inhibiting multiple endogenous signaling pathways
}

by

\author{
Nina Sofi Funa, ${ }^{1}$ Kristian Honnens de Lichtenberg, ${ }^{1,6}$ Maria Skjøtt Hansen, ${ }^{1,7}$ Jonas \\ van Cuyl Kuylenstierna, ${ }^{1,8}$ Kim Bak Jensen, ${ }^{1,2}$ Yi Miao, ${ }^{3,4,5}$ K. Christopher Garcia, ${ }^{3,4,5}$ \\ and Palle Serup ${ }^{1,9^{*}}$
}

${ }^{1}$ Novo Nordisk Foundation Center for Stem Cell Biology (DanStem), University of Copenhagen, DK-2200, Copenhagen, Denmark

${ }^{2}$ BRIC - Biotech Research and Innovation Centre, University of Copenhagen, DK2200, Copenhagen, Denmark

${ }^{3}$ Department of Molecular and Cellular Physiology, Stanford University School of Medicine, Stanford, CA 94305, USA

${ }^{4}$ Howard Hughes Medical Institute, Stanford University School of Medicine, Stanford, CA 94305, USA

${ }^{5}$ Department of Structural Biology, Stanford University School of Medicine, Stanford, CA 94305, USA

${ }^{6}$ Present address: Department of Stem Cell Discovery, Novo Nordisk A/S, DK-2760, Måløv, Denmark

${ }^{7}$ Present address: Department of Pediatrics and Cell \& Developmental Biology, Barbara Davis Center for Diabetes, University of Colorado Anschutz Medical Campus, Aurora, CO 80045, USA

${ }^{8}$ Present address: Department of Molecular Assays, Global Assay Development, Chr. Hansen A/S, Bøge Allé 10-12, DK-2970, Hørsholm, Denmark

${ }^{9}$ Lead contact

*Correspondence: palle.serup@sund.ku.dk 


\section{Summary}

2

3 Genetic differences between pluripotent stem cell lines causes variable activity of extra-cellular

4 signaling pathways, which limits the reproducibility of directed differentiation protocols. Here we

5 used human embryonic stem cells (hESCs) to interrogate how exogenously provided factors

6 modulate endogenous signaling events during specification of foregut endoderm lineages.

7 We find that TGF- $\beta 1$ activates an OTX2/LHX1 gene regulatory network that promotes anterior fate

8 by antagonizing endogenous Wnt signaling. In contrast to Porcupine inhibition, the effects of TGF- $\beta 1$

9 cannot be reversed by exogenous Wnt ligands, suggesting that induction of SHISA proteins and

10 intracellular accumulation of Fzd receptors make TGF- $\beta 1$ treated cells refractory to Wnt signaling.

11 Subsequently, TGF- $\beta 1$-mediated inhibition of Bmp- and Wnt-signaling suppresses liver- and

12 promotes pancreas fate. However, pancreas differentiation is delayed by TGF- $\beta 1$-induced CYP26A1

13 expression and inhibition of RA signaling. Our study thus identifies multiple mechanisms of crosstalk

14 between major developmental signaling pathways during foregut patterning.

16 Keywords Stem cells, Signaling, Patterning, TGF- $\beta$, Wnt, Bmp, RA, Pancreas, Liver 


\section{Introduction}

19 Directed differentiation of human embryonic stem cells (hESC) seeks to recapitulate the signaling events that govern cell lineage decisions, from germ layer specification during gastrulation, to subsequent regionalization along the anterior-posterior $(A-P)$ and dorsoventral (D-V) axes. In vertebrates, a combination of Wnt and high Nodal signaling triggers formation of the definitive endoderm (DE) layer that emerges from the primitive streak. Closure of the DE along the anterior- and caudal intestinal portals leads to formation of the primitive gut (PG). The PG is patterned along the A-P and D-V axes to form three broad regions: foregut, midgut and hindgut that are further patterned into distinct organ territories, including thyroid, lung, liver, pancreas and the different subdivisions of the gastro-intestinal tract (Arnold and Robertson, 2009; Grapin-Botton, 2005; Tam and Loebel, 2007). As is also the case for meso- and ectoderm, posterior endoderm fates are promoted by high levels of Wnt, BMP and retinoic acid (RA) signaling (Bayha et al., 2009; Deimling and Drysdale, 2009; Spence et al., 2011; Stevens et al., 2017), whereas ligand-sequestering antagonists such as Dkk1, Sfrp, Noggin and Chordin, secreted from anterior visceral endoderm and anterior mesendoderm (ME), protect anterior tissues from Wnt and BMP signaling and thus permit the development of anterior fates (McLin et al., 2007; Rankin et al., 2011). Similarly, the retinoic acid (RA) catabolizing enzyme, Cyp26a1, expressed in the anterior epiblast and the anterior half of the newly formed germ layers, protects anterior structures from RA exposure (Abu-Abed et al., 2001; Abu-Abed et al., 2003; Ribes et al., 2007). Later, these signaling pathways are repurposed and act in a stage-dependent manner to promote distinct organ lineages including liver and pancreas (Rankin et al., 2018). Liver and pancreas arise from common multipotent progenitors in the ventral foregut under the influence of FGF, BMP and Wnt signals emanating from adjacent, mesodermal tissues (Zaret, 2008). Dose-dependent FGF signaling from cardiogenic mesoderm and BMP-signaling from the septum transversum mesenchyme induces hepatic fate and suppresses pancreatic fate (Deutsch et al., 2001; Jung et al., 1999; Rossi et al., 2001; Serls et al., 2005). Accordingly, most $h E S C$ pancreas differentiation protocols use BMP signaling inhibitors to reduce liver specification (Nostro et al., 2011; Pagliuca et al., 2014; Rezania et al., 2014). Conversely, TGF- $\beta$ signaling has been shown to favor pancreatic over hepatic specification in hESC cultures (Loh et al., 2014). However, it is not clear how TGF- $\beta$ and BMP signaling interact during these fate decisions. 
How Wnt signaling acts on the multipotent foregut progenitors to specify organ fate is not fully understood (reviewed in Zaret, 2008). Ectopic Wnt signaling in Xenopus foregut progenitors inhibits the development of foregut organ buds, including pancreas, liver and lung, while repression of Wnt signaling cause an expansion of liver and pancreas buds (McLin et al., 2007). However, this can be interpreted as Wnt repression being required for specification of anterior endoderm, and thus foregut progenitors. In zebrafish, loss of canonical Wnt2/Wnt2bb signaling prevents liver and swim bladder specification and the pancreas and anterior intestines expand (Ober et al., 2006; Poulain and Ober, 2011). More recent studies in mouse and human systems indicate that non-canonical Wnt signaling is able to promote pancreatic over liver fate choice, while contradictory results were found regarding the ability of canonical Wnt signaling to promote liver fate (Mahaddalkar et al., 2020; Rodriguez-Seguel et al., 2013). Thus, the interaction between signals that regulate segregation of liver and pancreas primordia are still not fully understood. Similarly, we only have a limited understanding of how exogenously supplied growth factors affect endogenous signaling pathways at the various steps of hESC differentiation protocols. Although differences in endogenous signaling activity associated with different hESC lines (Ortmann et al., 2020) is an obvious source of variation in differentiation outcomes, the role of endogenous signaling pathways has not received a lot of attention. Generally, endogenously active pathways are inhibited by addition of antagonists, if considered detrimental to the desired differentiation outcome, often determined by trial-and-error approaches. Conversely, pathways whose activity is considered desirable are stimulated by addition of agonists. Yet, studies of embryonic development have uncovered numerous examples of crosstalk among signaling pathways and it is to be expected that variability of endogenous signaling, as well as addition of exogenous factors, will affect such crosstalk and thus the outcome of in vitro PSC differentiation in a cell line dependent manner. Here we investigated how exogenously provided growth factors and inhibitors affect endogenous signaling events during early pancreatic lineage specification in differentiating hESC cultures. By screening major developmental signaling pathways we identified TGF- $\beta 1$ and the porcupine inhibitor IWP-L6 as potent inducers of pancreatic fate when present during development of foregut endoderm and early pancreatic progenitors. We confirm the anteriorizing effect of Wnt inhibition and further show that TGF- $\beta 1$ anteriorizes endoderm by inhibiting the expression and/or function of components in the Wnt signaling pathway. 
Concurrently, TGF- $\beta 1$ and IWP-L6 both stimulate proliferation of OTX2 ${ }^{+}$anterior endoderm cells. We next show that $\mathrm{Wnt} / \beta$-catenin signaling promotes liver fate and suppress pancreas fate in hESC cultures and that TGF- $\beta 1$ suppresses liver fate by dual inhibition of BMP and Wnt signaling in foregut progenitors. Unexpectedly, we find that TGF- $\beta 1$-induced CYP26A1 inhibits RA signaling to delay pancreas specification and maintain foregut progenitors in an OTX2 ${ }^{+}$state.

Our work uncovers mechanisms that underlie an extensive crosstalk between developmental signaling pathways in foregut progenitors as they develop towards liver and pancreas during hESC differentiation. Knowledge about such signaling dynamics and their effect on progenitor proliferation will enable more efficient in vitro generation of insulinproducing beta cells.

Results

TGF- $\beta 1$ and Wnt inhibitors promote expression of pancreatic progenitor markers. When subjecting a PDX1 ${ }^{\mathrm{EGFP} /+}$ HUES4 reporter cell line (Ameri et al., 2017) to a control pancreas differentiation protocol (Rezania et al., 2014) we noted a disappointingly low expression of PDX1 at the end of stage 5 (S5), where endocrine differentiation commences (Figure $1 \mathrm{~A}$ and 1B). As induction of definitive endoderm (DE) appeared efficient at S1 (Figure S1A), we reasoned that subsequent anterior-posterior (A-P) patterning and/or specification of pancreatic endoderm (PE) was suboptimal in our cultures. We therefore added agonists or antagonists of selected pathways active in AP-patterning and pancreas specification to differentiating PDX1 ${ }^{\text {EGFP/+ }}$ HUES4 cells from S2 to S5 and monitored GFP expression at the end of S5 (D13). FACS analysis showed a significant increase in $\mathrm{GFP}^{+}$positive cells when blocking Wnt-secretion with the porcupine inhibitor IWP-L6 or adding either recombinant TGF- $\beta 1$ or Activin A, while perturbation of Notch signaling and relevant receptor tyrosine kinase pathways had only minimal effects (Figure 1B). Consistently, a prominent increase in

108 PDX1 protein expression was seen by immunofluorescent (IF) staining and western blotting 109 following treatment with IWP-L6 or TGF- $\beta 1$ (Figure S1B and S1D). Gene expression analysis

110 by qRT-PCR at D3-D13 showed that PDX1 and NKX6-1 transcripts increased from S3 and S5, 111 respectively, after IWP-L6 treatment, while the effect of TGF- $\beta 1$ on PDX1 expression was

112 only evident at S4. We also noted that expression of the anterior marker OTX2 was

113 significantly higher in both IWP-L6- and TGF- $\beta 1$-treated samples than in controls at S2 and 
114 S3. Conversely, expression of the posterior marker CDX2 and the early liver lineage marker

115 alpha fetoprotein (AFP) was suppressed from S2 and onwards by both treatments (Figure

116 1C). These results suggest that IWP-L6 inhibits the secretion of posteriorizing Wnt proteins

117 in our cultures, consistent with how Wnt proteins act in vivo (Loh et al., 2014; McLin et al.,

118 2007). This notion is supported by IWP-L6 being effective when added selectively at S2/3,

119 while later addition had no effect on pancreatic marker expression at S5 (Figure S1C).

120 Analysis of OTX2 protein expression confirmed a marked increase at S2 after treatment with

121 IWP-L6 or the Tankyrase inhibitor XAV-939, supporting that inhibition of Wnt signaling

122 anteriorizes the cells (Figure 1D and Figure S1D). Notably, TGF- $\beta 1$ treatment increased the

123 number of OTX2+ cells at S2 to the same extent as IWP-L6 (Figure 1D). Further IF analysis

124 showed an increase in PDX1 ${ }^{+}$cells already at S3 following IWP-L6 treatment and at S4

125 following TGF- $\beta 1$ treatment, both at the expense of $\mathrm{AFP}^{+}$cells that were present in

126 remarkably high numbers in control cells at both time points (Figure 1E and Figure S1D).

127 Taken together, these results suggest that endogenous Wnt proteins posteriorize hESC-

128 derived DE cultures while exogenous TGF- $\beta 1$ has the opposite effect.

TGF- $\beta 1$ anteriorize endoderm by antagonizing Wnt signaling. To gain a better understanding of how IWP-L6 and TGF- $\beta 1$ affect hESC-derived DE cultures, we performed RNA-seq analysis of cells treated with IWP-L6, TGF- $\beta 1$ or both at the end of S2 (D5) and S3 (D7) in the human ES cell lines HUES4 and H1 (Table S1). Principle component analysis (PCA) showed that the samples clustered according to both cell line and treatment. Importantly, the cells demonstrated a clear transcriptional response to the different treatments and the trajectories of the clusters along PC1 and PC2 were similar in both cell lines (Figure 1F). DESeq2 analysis found 92, 811 and 1417 genes differentially expressed (adjusted $p$-value < 0.05) in D5 cells treated with IWP-L6, TGF- $\beta 1$, or both, respectively, compared to control cells. Similarly, 732, 1481, and 1804 genes were differentially expressed at D7. 56, 484, and 717 genes were upregulated ( $\log 2 \mathrm{FC}>1)$ and 36, 327, and 700 genes were downregulated $(\log 2 \mathrm{FC}<-1)$ at D5. At D7 303, 791, and 911 genes were upregulated and 429, 690, and 1043 genes were downregulated (Table S2). Analysis by k-means clustering revealed that the majority of the regulated genes overlapped between the conditions (Figure 1G, $1 \mathrm{H}$ and

144 Table S2). Genes upregulated by TGF- $\beta 1$ and TGF- $\beta 1$ + IWP-L6 at D5 were found in clusters 1 and 6. Strikingly, IWP-L6 treatment also markedly upregulated cluster 1 genes, while cluster 
6 genes were weakly stimulated or unchanged (Figure $1 \mathrm{G}$ and Table S2). Cluster 1 and 6 included members of the gene regulatory network (GRN) responsible for anterior specification downstream of Smad2/3 such as EOMES, GSC, HHEX, LHX1 and OTX2 as well as downstream targets CER1, NOG, FZD5 and SHISA2 (from here on termed the OTX2/LHX1 GRN). Similarly, genes downregulated by TGF- $\beta 1$ and TGF- $\beta 1+$ IWP-L6 at D5 (clusters $2-5$ ) were typically also downregulated by IWP-L6, albeit more moderately. These included the posterior markers EVX1 and CDX2 as well as the Wnt and BMP agonists and target genes WNT11, RSPO3, LEF1, RNF43 and BMP5/6, BAMBI and ID2/4, respectively (Figure $1 \mathrm{G}$ and Table S2). Gene set enrichment analysis (GSEA) was dominated by enrichment of Gene Ontology (GO) categories relating cell cycle (see below), but also terms related to A-P pattern specification and negative regulation of Wnt signaling were enriched (Figure 1I, Table S3 and Table S4). Leading edge analysis of the latter two gene sets identified genes in the OTX2/LHX1 GRN, including OTX2, HHEX, LHX1, SIX3, FZD5 and CER1 (Figure 1J) and known Wnt pathway genes SHISA2, SFRP1/2, ROR2, TCF7L2, KREMEN2 following TGF- $\beta 1$ treatment (Figure 1K).

TGF- $\beta 1$ and IWP-L6 stimulate proliferation of anterior foregut progenitors. Addition of TGF- $\beta 1$ and/or IWP-L6 during S2-3 resulted in a marked increase in cell density from D5 and onwards (Figure 2A and 2F). Consistently, proliferation markers such as MKI67, PCNA, CCNA2, CCNB1, CDCA8, CDC20, CDC25A/B, E2F1, and MCM6 were upregulated at D5 by TGF- $\beta 1$ and IWP-L6 treatment (Figure 2B and Table S2). The top ten GO terms at D5 all related to processes involved in cell division and GSEA of IWP-L6 and/or TGF- $\beta 1$-treated D5 samples showed a significant enrichment of cell cycle related genes (Figure 2C, 2D, Table S3 and S4). We therefore assayed cell proliferation at D5 and D7 by EdU incorporation. An increase in proliferation was observed by FACS-analysis of EdU-incorporation at both timepoints (Figure 2E) and blocking TGF- $\beta$ signaling with the ALK4/5 inhibitor SB505124 significantly reduced EdU-incorporation at D7 (Figure 2F). The increase in EdU-percentage following IWP-L6 was also reversed with SB505124, indicating that TGF- $\beta 1$ and inhibition of Wnt-signaling might promote proliferation through a common mechanism. To test whether IWP-L6 and TGF- $\beta 1$ promote proliferation of bona fide PFG cells and not simply favor the 
177 at D5. IF analysis confirmed that both IWP-L6 and TGF- $\beta 1$ showed a significant increase in

178 the percentage of $\mathrm{OTX} 2^{+} \mathrm{EdU}^{+}$cells (Figure $2 \mathrm{G}$ and $2 \mathrm{H}$ ).

179 We next examined whether other growth factors present in the media during S2-3 were

180 providing proliferative cues under control conditions. We quantified EdU incorporation after

181 depletion of single factors from the control S2 and S3 media. We found that depletion of

182 FGF7, but not any other factor, from the media caused a two-fold decrease in the

183 percentage of EdU ${ }^{+}$cells at D5 and almost 10-fold reduction D7 (Figure 2I), consistent with

184 the role of mesenchymal FGF10 as a proliferative signal for multipotent pancreatic

185 progenitor cells in mice (Bhushan et al., 2001). We then tested whether FGF7 was required

186 for the proliferative effects of IWP-L6 and TGF- $\beta 1$. Strikingly, both IWP-L6 and TGF- $\beta 1$ was

187 capable of inducing proliferation in the absence of FGF7, albeit IWP-L6 was slightly less

188 potent compared to TGF- $\beta 1$ (Figure 2J).

189 As we found TGF- $\beta 1$ induced the OTX2/LHX1 GRN and OTX2 is able to directly activate cell

190 cycle genes (Bunt et al., 2012), we examined publicly available ATAC-seq and ChIP-seq data

191 for chromatin accessibility, Smad2 binding, and binding of selected TFs from the OTX2/LHX1

192 GRN to cell cycle genes in human ES cell cultures differentiating towards endoderm (Geusz

193 et al., 2021; Tsankov et al., 2015). This analysis revealed that cell cycle genes upregulated by

194 TGF- $\beta 1$ all showed binding of OTX2 in their promoter regions at the DE stage (Figure S2),

195 suggesting that OTX2 could be directly activating cell cycle genes in proliferating foregut

196 progenitors. Taken together, these findings suggest that Wnt-inhibition and TGF- $\beta 1$

197 stimulate proliferation of foregut progenitors via OTX2-mediated activation of the cell cycle.

TGF- $\beta 1$ activate an OTX2/LHX1 GRN that antagonize Wnt signaling at multiple levels. To better understand how TGF- $\beta 1$ antagonized Wnt signaling, we first analyzed expression of Wnt pathway agonists, antagonists and target genes in response to TGF- $\beta 1$ treatment. We noted high expression of WNT11 and moderate expression of WNT3, WNT5A and WNT5B in control cultures, while RSPO3 was the only R-Spondin expressed (Figure S3A and Table S1). Notably, TGF- $\beta 1$ treatment caused a significant reduction in expression of WNT11 and RSPO3 as well as the target genes LEF1 and RNF43, while similar reductions after IWP-L6 treatment only reached significance for $\angle E F 1$ and RSPO3 (Figure 3A). As expected, blocking TGF- $\beta 1$ action with SB431542 rescued expression of RSPO3 and LEF1 at D5, but had no 
both soluble and intracellular Wnt antagonists, including SFRP1, SHISA2 and SHISA3 at D5, while SFRP5 was suppressed. Similarly, we found induction of SFRP1, SFRP2, SHISA2 and SHISA4 at D7 (Figure 3B and Table S2). CER1 was also strongly induced at both timepoints, but may not antagonize Wnt signaling in humans (see discussion). Since TGF- $\beta 1$ treated D5 cells fail to activate Wnt target genes in response to exogenous Wnt3a/RSPO3 (Figure 3C), the induction of SHISA2/3 caught our attention as the encoded proteins antagonize Wnt signaling cell autonomously by sequestering Frizzled (Fzd) receptors in the endoplasmatic reticulum (ER) (Onishi and Zou, 2017; Yamamoto et al., 2005). We therefore analyzed Fzd mRNA and protein expression in control, IWP-L6 and TGF$\beta 1$-treated cultures. The four highest expressed Fzd transcripts were FZD4, $-5,-7$ and -8 (RPKM > 10; Figure S3A and Table S1). Consistent with mouse Fzd2, Fzd5, Fzd7 and Fzd8 being members of the Otx2/Lhx1 GRN (Costello et al., 2015; Sibbritt et al., 2018), treatment with IWP-L6 or TGF- $\beta 1$ increased FZD2 and FZD5 expression significantly, with smaller, nonsignificant increases in FZD7 and FZD8 (Figure S3C and Table S2). To visualize FZD receptors and to address whether TGF- $\beta 1$-induced SHISA2/3 expression correlated with FZD subcellular localization, we permeabilized control and TGF- $\beta 1$-treated cells and labeled them with a human-Fc-tagged version of the next-generation surrogate Wnt; DRPB-Fz7/8, which recognizes Fzd1, -2, -5, -7, and -8 (Dang et al., 2019; Miao et al., 2020). Confocal imaging showed prominent intracellular accumulation of FZD-receptors in TGF- $\beta 1$ treated samples compared to controls (Figure 3D), suggesting that SHISA2/3-mediated retention of FZD receptors in the ER may contribute to TGF- $\beta 1$-induced suppression of Wnt signaling. When examining Wht co-receptor expression we find a tendency to increased ROR2 mRNA in the RNA-seq data in response to IWP-L6 and TGF- $\beta 1$ (Figure S3D). We therefore analyzed ROR2 expression by qRT-PCR and found a twofold increase (Figure 3E). Conversely, LRP4 was downregulated (Figure S3D), while the more highly expressed LRP5/6 remained unchanged (Table S2). Overall, these changes may bias any residual Wnt signaling towards the non-canonical pathway.

Studies in mice, fish and frogs have found that genes encoding signaling antagonists such as Cer1, Sfrp1 and Shisa2 as well as the Wnt receptors Fzd2, Fzd5, Fzd7 and Fzd8 are activated by the concerted action of Otx2 and Lhx1 (Costello et al., 2015; Fossat et al., 2015; Sibbritt et al., 2018), while ligands such as Wnt8 and Wnt11 are repressed by Gsc in concert with Otx2 (Seiliez et al., 2006; Yao and Kessler, 2001; Yasuoka et al., 2014). Since these TFs were 
strongly induced by TGF- $\beta 1$ (Figure S3E), we assessed chromatin accessibility and TF binding

242 for these Wnt pathway genes in human ES cell cultures differentiating towards endoderm

243 (Geusz et al., 2021; Tsankov et al., 2015). This revealed enhanced chromatin accessibility

244 and binding of EOMES, FOXA2 and OTX2 at two putative cis-acting regions located 65 and

$245 \sim 130 \mathrm{~kb}$ downstream of the SHISA2 gene at the DE stage (Figure 3F). Data for human LHX1

246 were not available, but Otx2 and Lhx1 have previously been shown to bind the mouse

247 Shisa2 gene (Costello et al., 2015). Similarly, two regions located $\sim 30$ and $\sim 117 \mathrm{~kb}$

248 downstream of the SHISA3 gene and multiple regions within an $\sim 80 \mathrm{~kb}$ region upstream of

249 SFRP1 showed enhanced chromatin accessibility at the DE stage and binding of EOMES,

250 FOXA2 and OTX2, while CER1 showed enhanced chromatin accessibility and binding of

251 SMAD2, EOMES, FOXA2 and OTX2 at the promoter region at both ME and DE stages (Figure

252 3F). Taken together, these findings suggest that TGF- $\beta 1$ treatment at S2 acts by maintaining

253 expression of a conserved OTX2/LHX1/GSC GRN that suppresses Wnt/ $\beta$-catenin signaling via

254 multiple mechanisms including ligand downregulation, induction of antagonists, and

255 changes in co-receptor expression.

TGF- $\beta 1$ induced inhibition of BMP- and Wnt-signaling promotes pancreas over liver specification. The strong suppression of AFP expression by IWP-L6 and TGF- $\beta 1$ prompted us to further investigate pancreas versus liver differentiation in our cultures. In addition to AFP, other early liver-specific genes such as, APOA1, FABP1 and TTR were all reduced at D7

261 (Figure 4A). Furthermore, GSEA of the RNA-seq datasets showed that both IWP-L6 and TGF-

$262 \beta 1$ strongly reduced expression of liver specific genes (Figure 4B and S4A) and hepatocyte signatures were the most reduced among single cell RNA-seq-based cell type signatures during S2-3 (Figure S4B, Table S4 and S5). Furthermore, GSEA of our RNA-seq data compared to RNA-seq data from LASER-capture micro dissected dorsal pancreatic buds (DP) and hepatic cords (HC) of Carnegie stage 13 human embryos (Jennings et al., 2017) confirmed that expression of genes that were induced or reduced in IWP-L6-treated cells D7 corresponded to genes seen in the dorsal pancreas (DP high) and hepatic cord (HC high) gene sets, respectively (Figure 4C, S4C and Table S6). Notably, IWP-L6 or TGF- $\beta 1$ treatment at S2 alone (D4-5) was sufficient to suppress AFP at D7, indicating that Wnt and TGF- $\beta$

271 signaling modulate lineage segregation between liver and pancreas already at the primitive 
gut tube stage (Figure S4D). Taken together, these results indicate that canonical Wnt signaling promotes human liver specification, as it does in zebrafish (Ober et al., 2006; Poulain and Ober, 2011) and that TGF- $\beta$ signaling has an opposing effect.

To understand how TGF- $\beta$ signaling inhibits liver specification we interrogated our RNA-seq data for potential changes in BMP and Wnt signaling, two known liver promoting pathways. First, we found that the classical BMP ligands $B M P 2, B M P 4, B M P 5, B M P 6$ and $B M P 7$ were expressed at moderate to high levels in control conditions (Figure S4E). TGF- $\beta 1$ significantly suppressed expression of BMP5 and BMP6 and strongly induced the BMP antagonists CER1, FST and NOG (Figure 4D and S2E). In contrast, IWP-L6 had no effect on these genes with the exception of BMP5. Consistently, the downstream target genes ID2, ID4 and BAMBI were downregulated by TGF- $\beta 1$ but not by IWP-L6 (Figure 4E). Together, these findings suggest that TGF- $\beta 1$-induced suppression of liver markers may be mediated, at least partly, through suppression of BMP signaling. To test to whether reduced Wnt ligand availability might account for the IWP-L6- and TGFß1-mediated suppression of liver markers, we co-treated cells with recombinant Wnt3a and/or RSPO3 during S2-3 and assayed AFP and FABP1 expression. As expected, IWP-L6mediated inhibition of liver markers D7 was fully reversed by co-treatment with Wnt3a with or without additional RSPO3 but not by RSPO3 alone (Figure 4F and 4G). However, Wnt3a, with or without RSPO3, did not prevent TGF- $\beta 1$-induced inhibition of AFP and FABP1 expression, indicating that suppression of Wnt ligand expression cannot fully account for the effect of TGF- $\beta 1$ on liver differentiation seen at D7. As also seen at D5, treatment with Wnt3a and RSPO3 failed to induce expression of Wnt target genes in the presence of TGF- $\beta 1$

294 (Figure 4H), showing that TGF- $\beta 1$ blocks Wnt-signaling downstream of ligand availability.

295 This may be due to increased expression of the antagonists SFRP1/2 and SHISA2/4 at D7

296 (Figure 3B).

297 Lastly, we asked whether BMP signaling influenced TGF- $\beta 1$-mediated suppression of Wnt 298 signaling and liver differentiation. Notably, BMP-signaling increased expression of Wnt11 299 and Wnt downstream targets LEF1, RSPO3 and RNF43, and both IWP-L6- and TGF- $\beta 1$ -

300 mediated suppression of these genes at D7 was partially rescued by replacing LDN193189 with BMP4 (Figure 4I). Strikingly, omitting the BMP receptor inhibitor LDN193189 and/or adding exogenous BMP4 or BMP6 counteracted IWP-L6- and TGF- $\beta 1$-induced suppression of 
304 4J-L). These data suggest that TGF- $\beta 1$-mediated suppression of BMP signaling may contribute to reduced Wnt signaling by attenuating Wnt ligand expression.

TGF- $\beta 1$ delays pancreatic differentiation by attenuating RA signaling. To begin to unravel the mechanism responsible for the delayed induction of PDX1 following TGF- $\beta 1$ treatment we interrogated our RNA-seq datasets for changes in signaling pathways known to be important for PDX1 gene expression. We noted a strong induction of CYP26A1 expression at

311 D5 in TGF- $\beta 1$ treated cells, which was augmented by IWP-L6 co-treatment, while IWP-L6

312 alone had no effect (Figure 5A). At D7 CYP26A1 expression was high regardless of TGF- $\beta 1$

313 addition, most likely due to the presence of $1 \mu \mathrm{M}$ RA in S3 medium (Rezania et al., 2014).

314 Nevertheless, we did observe reduced expression of ALDH1A1 (encoding the RA-

315 synthesizing enzyme RALDH1) at D7 in response to TGF- $\beta 1$ treatment (Figure 5A). These

316 data suggest that TGF- $\beta 1$-treatment during S2 might desensitize the cells to RA added at S3.

317 To test this hypothesis, we examined expression of pancreatic markers in control, TGF- $\beta 1$

318 and IWP-L6-treated cells at D7, after co-treatment with the CYP26A1-inhibitor, R115866 at

319 S2 (Figure 5B). Notably, addition of R115866 during S2 prevented the TGF- $\beta 1$-induced

320 suppression of PDX1, SOX9, ONECUT1 and HNF1B expression and blunted the induction of

321 OTX2 expression at D7 (Figure 5C). However, inhibition of CYP26A1 did not prevent TGF- $\beta 1$ -

322 mediated suppression of AFP expression. The effect of R115866 on OTX2 and PDX1

323 expression was also evident on the protein level (Figure 5D and 5E). To begin to understand

324 how anterior CYP26A1 expression is activated we again examined publicly available ATAC-

325 seq and ChIP-seq data for chromatin accessibility and binding of Smad2 and TFs from the

326 Otx2/Lhx1 GRN to the CYP26A1 locus. This analysis revealed regions of increased chromatin

327 accessibility at D3 where cells have been exposed to Activin A and again at D7 where cells

328 have been exposed to RA (Figure 5F). Notably, the immediate 5 '-flanking region was bound

329 by SMAD2 and EOMES at the ME stage and by OTX2 at the DE stage (Figure 5F). Taken

330 together, these results suggest that TGF- $\beta 1$ signaling delays pancreatic differentiation via

331 SMAD2-induced CYP26A1 expression, which desensitizes foregut cells to RA exposure. 


\section{Discussion}

334 Here we show that TGF- $\beta$ signaling anteriorize hESC-derived definitive endoderm and subsequently promote pancreas fate over liver ditto via inhibition of Wnt/ $\beta$-catenin and Bmp signaling in foregut progenitors. Our results uncover mechanisms used by TGF- $\beta$ to modulate the activity of other signaling pathways operating in differentiating hESCs. We observed a very similar response to TGF- $\beta 1$ and the Porcupine inhibitor IWP-L6, for which the most parsimonious explanation is suppression of Wnt signaling by TGF- $\beta 1$. Remarkably, we also noted a strong stimulation of proliferation of S2 and S3 progenitors with these treatments. The exact mechanism still needs to be elucidated, but may involve direct activation of cell cycle genes by OTX2. Notably, the proliferative effect is independent of exogenous FGF. Together these observations should prove useful in relation to improving the efficacy of directed differentiation protocols for hESCs. TGF- $\beta /$ Nodal-induced Smad2/3 signaling promotes anterior fate in mice by inducing expression of Eomes that directly activates Foxa2 and the LIM domain homeobox transcription factor Lhx1, which in partnership with Otx2, activates numerous anterior ME genes as well as negative regulators of Bmp-, Nodal-, and Wnt/ $\beta$-catenin signaling (Costello et al., 2015; Fossat et al., 2015; Ip et al., 2014; Sibbritt et al., 2018). We observed strong, induction of EOMES, GSC, LHX1 and OTX2 and other anterior TFs after TGF- $\beta 1$ treatment at S2, indicating anteriorization of the endoderm formed at S1. Many of these markers were also induced by IWP-L6 treatment, as expected when inhibiting the posteriorizing effect of Wnt signaling. Together, these observations are best explained by suppression of Bmp-, Nodal and Wnt-signaling. This suppression appears to occur at several levels including repression of ligand expression and activation of antagonists such as CER1, FST, LEFTY1/2, SFRP1 and SHISA2/3. The antagonists are well known targets of the Otx2/Lhx1 GRN in mouse and Xenopus (Costello et al., 2015; Fossat et al., 2015; Sibbritt et al., 2018) and our analysis of published ChIP-seq data (Tsankov et al., 2015) indicate that this may also be the case in humans. Future ChIP-seq analysis of LHX1 in human cells could bolster this notion.

360 The mechanism underpinning the observed suppression of BMP and WNT ligand expression is unresolved, but GSC is a strong candidate as a direct repressor of ligand genes as it is induced by TGF- $\beta 1$ in our cells and serve such a role in vivo, in concert with OTX2 (Yasuoka et al., 2014). Indeed, this study suggests that co-binding of OTX2 with either LHX1 or GSC 
anterior development. SIX3, which in addition to anterior neural plate is expressed in hESCderived ME and in early mouse DE (Shim et al., 2020), may also be involved. It is induced, albeit at low levels, by TGF- $\beta 1$ in our cells and has been shown to repress BMP and WNT ligand expression in anterior neural plate (Gestri et al., 2005; Lagutin et al., 2003). Again, future ChIP-seq analyses of GSC and SIX3 in hESC-derived DE will help test these notions. The identity of the endogenous Wnt ligand(s) that act as posteriorizing factors in our cultures is not fully resolved by our data. However, we propose that Wnt11 is the key ligand for several reasons. First, Wnt11 is by far the highest expressed ligand in our culture with 610-fold higher RPKM values than any other Wnt ligand. Second, we find that Wnt11, together with RSPO3, is strongly suppressed by TGF- $\beta 1$ treatment. Third, Wnt11 has been shown to be involved in foregut patterning in Xenopus, where Wnt11 activity must be suppressed by Sfrp5 in the anterior endoderm in order to maintain anterior foregut endoderm identity (Li et al., 2008; McLin et al., 2007). These studies found Sfrp5 expressed in surface cells of the foregut epithelium, in close proximity to the deep endoderm that expresses Wnt11, which activated both the Wnt/ $\beta$-catenin and the Wnt/PCP pathways. Notably, morpholino-mediated depletion of Sfrp5 or Wnt11 overexpression in Xenopus embryos both caused a loss of foregut identity.

Looking at organ-specific markers, we found that liver markers were suppressed by both TGF- $\beta 1$ and IWP-L6 and this was evident from D5 and onwards. Conversely, differentiation towards the pancreatic lineage was promoted, but only from D10 and onwards after TGF- $\beta 1$ treatment. Analysis of endogenous signaling activity based on expression of well-known target genes at D5 and D7 revealed the expected suppression of Wnt signaling by IWP-L6 and suppression of both Wnt and Bmp signaling in response to TGF- $\beta 1$. The TGF- $\beta$ and BMP pathways often cross repress each other, through mechanisms that are not fully resolved, but may in some cases involve sequestration of a limited pool of Smad4 (Candia et al., 1997; Galvin et al., 2010). A reciprocal relationship between TGF- $\beta$ and BMP in relation to induction of liver versus pancreas lineages has previously been reported in hESCs (Loh et al., 2014), but a mechanism was not described. Our results are thus consistent with previous observations and our RNA-seq data suggest that TGF- $\beta 1$ may suppress Bmp signaling in foregut progenitors by repressing BMP6 expression and inducing Bmp antagonists such as NOG, FST and CER1. CER1 was strongly induced at both D5 and D7, while NOG and FST were 
induced at D5 and D7, respectively. While Bmp signaling is well known to promote liver development in vivo (Chung et al., 2008; Rossi et al., 2001), a mechanism involving suppression of BMP signaling may still appear surprising at first glance as the Bmp receptor inhibitor LDN193189 is present in S3 medium. However, as LDN193189 is only added at S3, it may leave time for endogenously produced BMPs to act at S2. Furthermore, LDN193189 only inhibits BMPR1B 50\% at the concentration used here (Sanvitale et al., 2013).

Notably, we found that blocking Wnt secretion via Porcupine inhibition promoted pancreas differentiation, while suppressing liver differentiation and that TGF- $\beta 1$ also suppressed Wnt signaling at D7. In zebrafish, the ligands Wnt2 and Wnt2bb act via Fzd5 to activate Wnt/ $\beta$ catenin signaling and promote liver development (Ober et al., 2006; Poulain and Ober, 2011), but whether and how Wnt/ $\beta$-catenin signaling promotes mammalian liver development has been less clear. Non-canonical Wnt signaling has been reported to favor pancreas over liver fate, but no evidence of Wnt/ $\beta$-catenin signaling promoting liver fate was found in the same study (Rodriguez-Seguel et al., 2013). However, recent work on hESC-derived foregut progenitors did find that Wnt/ $\beta$-catenin signaling promoted liver fate (Mahaddalkar et al., 2020), and in agreement with this finding, we could rescue liver fate in IWP-L6 treated cultures by addition of Wnt3a. In our hESC cultures, the two most highly expressed WNT genes are WNT5A and WNT11, making these candidates for inducing liver fate, likely augmented by RSPO3. Like Wnt5a, Wnt11 is often considered a non-canonical ligand but both ligands can also activate the canonical pathway if the responding cells express the proper receptors (Li et al., 2008; Mikels and Nusse, 2006; Tao et al., 2005). However, the identity of a putative liver specifying Wnt ligand in vivo remains unknown, but candidates are Wnt2, Wnt2b and Wnt5a, which are all expressed in the mesoderm proximal to the developing liver (McMahon and McMahon, 1989; Monkley et al., 1996; RodriguezSeguel et al., 2013; Zakin et al., 1998). Mouse Wnt2/Wnt2b double mutants display normal liver and pancreas development (Goss et al., 2009), but additional Wnt ligands (e.g. Wnt5a) have been suggested to fulfill the liver specifying role in mammals (Poulain and Ober, 2011). Notably, the canonical co-receptor Lrp5 is enriched in liver progenitors compared to pancreas further suggesting that $\mathrm{Wnt} / \beta$-catenin signaling may promote liver fate. $\mathrm{A}$ 
progenitors and, as mentioned above, non-canonical signaling favors pancreas over liver fate (Rodriguez-Seguel et al., 2013).

As also seen at D5, TGF- $\beta 1$ suppressed Wnt activity at D7 even in the presence of exogenous Wnt ligand, possibly by stimulating SFRP1/2 and SHISA2/4 expression at D7. CER1 is also strongly induced, but human CER1, which is $69 \%$ identical to mouse Cer1, may not antagonize Wnt signaling (Belo et al., 2000; Piccolo et al., 1999). Importantly, omitting LDN193189 from in the S3 medium, or replacing it with Bmp ligand, reactivated WNT11and Wnt target gene expression and partially restored liver differentiation in the presence of TGF- $\beta 1$. This suggests that Bmp signaling acts upstream of Wnt signaling and that TGF- $\beta 1$ induced suppression of Wnt signaling could be mediated, at least partly, by inhibition of Bmp stimulated Wnt activity. Consistent with this notion, Bmp2b acts prior to Wnt2bb and Wnt2 in zebrafish liver development (Chung et al., 2008; Poulain and Ober, 2011). Taken together, our findings show that BMP- and Wnt/ $\beta$-catenin signaling coordinate induction of liver lineage in human progenitors and suggest mechanisms for TGF- $\beta 1$-mediated suppression of both pathways that ultimately promotes pancreatic over liver fate.

One notable difference between the effect of TGF- $\beta 1$ and IWP-L6 was the delay in onset of pancreatic marker expression in TGF- $\beta 1$ treated samples. Intriguingly, a previous study found suppression of Pdx1 expression in the developing pancreas after treatment of 3-4 and 5-6 somite stage half-embryo cultures with TGF- $\beta 2$ and an increase after treatment of 5-6S half-embryo cultures with the ALK4,5,7 inhibitor SB431542. The underlying mechanism was not elucidated, but it was shown that changes in proliferation rate or apoptosis were not involved. (Wandzioch and Zaret, 2009). Importantly, our RNA-seq data showed that addition of TGF- $\beta 1$ during $\mathrm{S} 2$ induced a profound increase in the expression of the RA-degrading enzyme CYP26A1. This induction is likely a reflection of the normal anterior expression pattern observed for Cyp26a1 in mouse embryos (Abu-Abed et al., 2001; Abu-Abed et al., 2003; Ribes et al., 2007), and may be a direct induction by SMAD2, possibly in conjunction with EOMES. As RA is well-known inducer of Pdx1 and pancreatic fate (Kraus and GrapinBotton, 2012; Micallef et al., 2005; Molotkov et al., 2005; Stafford and Prince, 2002), elevated CYP26A1 levels could explain the delay in the onset of pancreatic marker expression. Indeed, the delay was prevented by adding the CYP26A1 inhibitor R115866. This

460 finding may partly explain why many pancreas differentiation protocols rely on ALK4,5,7 
461 inhibitors during later stages (Nostro et al., 2011; Pagliuca et al., 2014; Rezania et al., 2014;

462 Rezania et al., 2011).

463 In summary, our study suggests several mechanisms by which Bmp, TGF- $\beta$ and Wnt signaling 464 interact to control differentiation of hESC-derived foregut progenitors. Our findings improve

465 the understanding of human foregut patterning and organ development and provide

466 valuable new avenues for improving directed differentiation protocols for hESCs in order to

467 obtain clinically relevant cell types in unlimited quantities.

Acknowledgements. We thank Silvia Raineri for bioinformatics assistance and Jutta 470 Bulkescher, Gelo de la Cruz, Paul van Dieken, Helen Neil, and Magali Michaut and the DanStem Research Platforms for technical assistance and the use of instruments. This work was supported by the Juvenile Diabetes Research Foundation International (3-APF-2017-390-

473 A-N), the European Commission's $7^{\text {th }}$ Framework Programme for Research (agreement 602587), the NIH (R01DK115728), Howards Hughes Medical Institute and Mathers Foundation. The Novo Nordisk Foundation Center for Stem Cell Biology is supported by grant number NNF17CC0027852.

Author Contributions. N.S.F. and P.S. conceived the study, designed and interpreted experiments and wrote the manuscript. N.S.F, M.S.H. and J.v.C.K. carried out experiments, while N.S.F and K.H.L. performed the bioinformatics analyses. K.B.J., Y.M. and K.C.G. designed and produced the NGS detection reagent. All authors revised and approved the manuscript.

Declaration of Interests. The authors declare no competing interests.

\section{Figure legends}

Figure 1. TGF- $\beta 1$ and IWP-L6 anteriorize endoderm and promote pancreas over liver fate

487 (A) Schematic overview of the basic differentiation protocol. The stages when compounds 488 being screened were added are indicated. DE: definitive endoderm; PGT: primitive gut tube; 489 PP1: pancreatic progenitor 1; PP2: pancreatic progenitor 2; EP: endocrine precursor.

490 (B) FACS analyses of PDX1 ${ }^{\mathrm{EGFP} /+}$ HUES4 cells showing percent $\mathrm{GFP}^{+}$cells at D13 after

491 treatment with indicated factors during S2-S5. Mean $\pm S D, N=3 . * p<0.05, * * p<0.005$, 492 $* * * * \mathrm{p}<0.0001$. 
(C) qRT-PCR analyses of $O T X 2, C D X 2, A F P, P D X 1$ and NKX6-1 expression in differentiating

494 HUES4 cells at the indicated time points after treatment with the indicated factors during S2-S3. Data are shown relative to undifferentiated HUES4 cells. Mean $\pm S D, N=3, * p<0.05$, $* * \mathrm{p}<0.005, * * * \mathrm{p}<0.0005, * * * * \mathrm{p}<0.0001$.

497 (D) IF staining for OTX2 in differentiating HUES4 cells at D5 after treatment with the

498 indicated factors during S2. The cells were counterstained with DAPI. Scale bar, $50 \mu \mathrm{m}$.

499 (E) IF staining for PDX1 (green) and AFP (red) in differentiating HUES4 cells at D7 and D10

500 after treatment with the indicated factors during S2-S3. The cells were counterstained with

501 DAPI. Insets show higher magnifications of boxed areas. Scale bars, $50 \mu \mathrm{m}$.

502 (F) Principle component analysis (PCA) showing clustering along PC1 and PC2 for PDX1 $1^{\mathrm{EGFP} /+}$

503 HUES4 $(n=3)$ and H1 $(n=2)$ cells treated with IWP-L6, TGF- $\beta 1$ or IWP-L6 + TGF- $\beta 1(I+T)$ during

504 S2 (D5) or S2-S3 (D7).

505 (G) Heatmaps scaled by row to show patterns of regulated genes in PDX1 ${ }^{\text {EGFP/+ }}$ HUES4 cells

$506(n=3)$ by k-means clustering analysis of RNA-seq data. Signature genes are indicated for each 507 cluster.

$508(\mathrm{H})$ Venn diagram showing overlap of all deregulated genes at D5 and D7 with an FDR $<0.1$ 509 and fold change $>1.5$.

510 (I) GSEA plots comparing gene expression data from each treatment at D5 ( $n=3)$ with the

511 indicated gene sets. Normalized Enrichment Scores (NES) and p-values are indicated.

512 (J-K) GSEA plots comparing D5 gene expression data from each treatment (J) or TGF- $\beta 1$ (K)

513 with gene sets for anterior-posterior pattern specification or negative regulation of Wnt

514 signaling, respectively $(\mathrm{N}=3)$. Signature genes from leading and trailing edge analyses are

515 shown in red and blue boxes, respectively. Normalized Enrichment Scores (NES) and p-

516 values are shown.

$517 \quad$ See also Figure S1.

518

519 Figure2. TGF- $\beta 1$ and IWP-L6 stimulate proliferation of anterior foregut progenitors

520 (A) DAPI staining showing increased cellular density at D7 and D10 following treatment with 521 IWP-L6 or TGF- $\beta 1$ during S2-S3. Scalebar, $100 \mu \mathrm{m}$. 
522 (B) Expression of cell cycle related genes by RNA-seq in PDX1 ${ }^{\mathrm{EGFP} /+}$ HUES4 cells treated with 523 IWP-L6, TGF- $\beta 1$ or IWP-L6 + TGF- $\beta 1$ (I+T) during S2 (D5) or S2-S3 (D7). Mean \pm SEM, N=3, * $524 \operatorname{padj}<0.05, * *$ padj $<0.005, * * *$ padj $<0.0005, * * * * \operatorname{padj}<0.0001$.

525 (C) NES-scores for the ten most enriched biological process GO terms for genes enriched at 526 D5 in cells treated with IWP-L6 or TGF- $\beta 1$ during S2.

527 (D) GSEA plots comparing gene expression data from each treatment at D5 with a gene set 528 for cycling genes. Normalized Enrichment Score (NES) and p-values are indicated.

529 (E) FACS analysis of EdU incorporation and DNA content in differentiated $P D X 1^{\mathrm{EGFP} /+}$ HUES4 530 cells at D5 and D7 after treatment with vehicle, IWP-L6 or TGF- $\beta 1$ during S2 (D5) or S2-S3 531 (D7).

532 (F) Percentage of EdU ${ }^{+}$cells in differentiated PDX1 $1^{\mathrm{EGFP} /+}$ HUES4 cells at D5 and D7 after 533 treatment with the indicated factors. Mean $\pm \mathrm{SD}, \mathrm{N}=3, * \mathrm{p}<0.05, * * \mathrm{p}<0.005, * * * * \mathrm{p}<$ 5340.0001.

535 (G) IF analysis of OTX2 $/ \mathrm{EdU}^{+}$co-expression following treatment with vehicle, IWP-L6 or 536 TGF- $\beta 1$. Scalebar, $100 \mu \mathrm{m}$.

537 (H) Quantification of OTX2+/EdU ${ }^{+}$co-expression following treatment with vehicle, IWP-L6 or 538 TGF- $\beta 1$. Mean \pm SD, N=3, $* p<0.05, * * p<0.005, * * * * p<0.0001$.

539 (I) Percentage of $\mathrm{EdU}^{+}$cells following depletion of individual factors from the control

540 medium. Mean $\pm \mathrm{SD}, \mathrm{N}=3, * \mathrm{p}<0.05, * * \mathrm{p}<0.005, * * * \mathrm{p}<0.0005$.

541 (J) Percentage of EdU ${ }^{+}$cells following withdrawal of FGF7 in cells treated with IWPL6 or TGF-

$542 \beta 1$. Mean $\pm S D, N=3, * p<0.05, * * p<0.005, * * * p<0.0005, * * * p<0.0001$.

543 See also Figure S2.

545 Figure3. TGF- $\beta 1$ activates an OTX2/LHX1 GRN that antagonize Wnt signaling

546 (A) Expression of WNT11, RSPO3, LEF1 and RNF43 by RNA-seq in PDX1 ${ }^{\text {EGFP/+ }}$ HUES4 cells 547 treated with vehicle (Ctrl), IWP-L6, TGF- $\beta 1$ or IWP-L6 + TGF- $\beta 1$ (I+T) during S2 (D5) or S2-S3

548 (D7). Mean $\pm \mathrm{SEM}, \mathrm{N}=3, * \operatorname{padj}<0.05, * * \operatorname{padj}<0.005, * * * * \operatorname{padj}<0.0001$.

549 (B) Differential expression of Wnt-signaling antagonists by DESeq2 analysis of RNA-seq data 550 from PDX1 ${ }^{\text {EGFP/+ }}$ HUES4 cells treated with TGF- $\beta 1$ or IWP-L6 + TGF- $\beta 1$ during S2 (D5) or S2-S3 551 (D7). Log2 fold change $(\log 2 F C)$ relative to vehicle controls is shown as are adjusted $p$-values 552 (padj). 
(C) qRT-PCR analysis of LEF1, RNF43 and RSPO3 expression at D5 in response to Wnt3a +

554 RSPO3 stimulation during S2 in control cells (Ctrl) or cells treated with IWP-L6 or TGF- $\beta 1$.

555 Mean $\pm S D, N=3, * p<0.05$.

556 (D) Confocal microscopy of D5 PDX1 ${ }^{\mathrm{EGFP} /+}$ HUES4 cells stained for $\beta$-catenin (green) and Fzd

557 receptors (red) after treatment with vehicle (Ctrl) or TGF- $\beta 1$ during S2.

558 (E) qRT-PCR analysis of ROR2 expression at D5 in PDX1 ${ }^{\mathrm{EGFP} /+}$ HUES4 cells treated with IWP-

559 L6, TGF- $\beta 1$ or IWP-L6 + TGF- $\beta 1(I+T)$ during S2. Mean \pm SD, $N=3, * p<0.05$.

560 (F) Signal tracks of ATAC-seq from Geusz et al. (2021) and SMAD2, EOMES, FOXA2 and OTX2

561 ChIP-seq data from Tsankov et al. (2015) at the SHISA2, SHISA3, SFRP1 and CER1 loci.

562 Dashed lines are used to mark areas with differential chromatin accessibility at the DE stage,

563 and TF binding at the ME and/or DE stages. ES: ES cells; DE: definitive endocerm; GT:

564 primitive gut tube; PP1: pancreatic progenitor 1, ME: mesendoderm.

565 See also Figure S3.

566

Figure 4. TGF- $\beta 1$ promotes pancreas over liver fate by inhibiting BMP- and Wnt-signaling

(A) Expression of AFP, APOA1, FABP1 and TTR by RNA-seq in PDX1 ${ }^{\mathrm{EGFP} /+}$ HUES4 cells treated with IWP-L6, TGF- $\beta 1$ or IWP-L6 + TGF- $\beta 1$ (I+T) during S2 (D5) or S2-3 (D7). Mean $\pm \mathrm{SEM}, \mathrm{N}=3$, $* \operatorname{padj}<0.05, * * \operatorname{padj}<0.005, * * * * \operatorname{padj}<0.0001$.

(B) GSEA plots comparing gene expression data from each treatment at D7 with a gene set

572 for liver specific genes (Hsiao et al., 2001). Normalized Enrichment Score (NES) and p-values 573 are shown.

574 (C) GSEA plots comparing gene expression data from each treatment at D7 with genes

575 highly expressed in human Carnegie Stage (CS)13 dorsal pancreas (DP) vs hepatic cords (HC)

576 and genes highly expressed in HC vs DP (Jennings et al., 2017).

577 (D-E) Expression of BMP ligands, secreted BMP antagonists (D) and BMP target genes (E) by

578 RNA-seq in PDX1 ${ }^{\text {EGFP/+ }}$ HUES4 cells treated with IWP-L6, TGF- $\beta 1$ or IWP-L6 + TGF- $\beta 1$ (I+T)

579 during S2 (D5) or S2-S3 (D7). Mean \pm SEM, N=3, ** padj < 0.005, *** $\operatorname{padj}<0.0005, * * * *$

580 padj $<0.0001$.

581 (F) IF analysis of $\mathrm{AFP}^{+}$cells at D7 in response to Wnt3a, RSPO3 or Wnt3a + RSPO3

582 stimulation during S2-S3 in cells treated with vehicle, IWP-L6 or TGF- $\beta 1$. 
$583(\mathrm{G}-\mathrm{H})$ qRT-PCR analysis of $A F P, F A B P 1(\mathrm{G}), L E F 1$ and $R S P O 3(\mathrm{H})$ expression at $\mathrm{D} 7$ in response

584 to Wnt3a, RSPO3 or Wnt3a + RSPO3 stimulation during S2-S3 in PDX1 ${ }^{\mathrm{EGFP} /+}$ HUES4 cells

585 treated with vehicle (Veh), IWP-L6 or TGF- $\beta 1$. Mean \pm SD, N=3, $* p<0.05$.

586 (I) Expression of WNT11, LEF1, RSPO3 and RNF43 by qRT-PCR in response to replacement of

587 LDN193189 with BMP4 during S3 in PDX1 ${ }^{\mathrm{EGFP} /+}$ HUES4 cells treated with vehicle (Veh), IWP-

588 L6 or TGF- $\beta 1$. Mean \pm SD, N=3, $* p<0.05, * * p<0.005$.

589 (J) IF analysis of $\mathrm{AFP}^{+}$cells at D7 in response to replacement of LDN193189 with BMP6

590 during S3 in PDX1 ${ }^{\mathrm{EGFP} /+}$ HUES4 cells treated with vehicle (Veh), IWP-L6 or TGF- $\beta 1$.

591 (K) Western blot showing AFP and PDX1 expression in PDX1 ${ }^{\mathrm{EGFP} /+}$ HUES4 cells cultured in

592 standard S3 medium (Ctrl), S3 medium without LDN193189 (w/o LDN) or with replacement

593 of LDN193189 with either BMP4 or BMP6 during S3, in cells treated with vehicle (Veh), IWP-

594 L6 (IWP) or TGF- $\beta 1$ (TGF). Also shown are hESC and DE stages. Vinculin is used as internal

595 control.

596 (L) Expression of AFP and FABP1 by qRT-PCR in standard S3 medium (Ctrl), S3 medium

597 without LDN193189 (w/o LDN) or with replacement of LDN193189 with BMP4 during S3, in

598 cells treated with vehicle (Veh), IWP-L6 or TGF- $\beta 1$. Mean \pm SD, N=3, $* p<0.05, * * p<0.005$.

599 See also Figure S4.

600

601 Figure 5. TGF- $\beta 1$ delays pancreatic differentiation by attenuating RA signaling

602 (A) Expression of CYP26A1 and ALDH1A1 measured by RNA-seq in PDX1 ${ }^{\text {EGFP/+ }}$ HUES4 cells

603 treated with IWP-L6, TGF- $\beta 1$ or IWP-L6 + TGF- $\beta 1$ (I+T) during S2 (D5) or S2-S3 (D7). Mean \pm

604 SEM, N=3, ** padj $<0.005$ and $* * * *$ padj $<0.0001$.

605 (B) Schematic overview of protocol using a CYP26-selective inhibitor, R115866 with TGF- $\beta 1$.

606 (C) Expression of PDX1, SOX9, ONECUT1, HNF1B, OTX2 and AFP measured by qRT-PCR in D7

$607 P D X 1^{\mathrm{EGFP} /+}$ HUES4 cells treated with vehicle (DMSO), TGF- $\beta 1$ and/or R115866 as indicated.

608 (D) Western blot showing OTX2, PDX1 and AFP expression in D7 PDX1 ${ }^{\mathrm{EGFP} /+}$ HUES4 cells

609 treated with vehicle (Veh), IWP-L6, TGF- $\beta 1$ and/or R115866 as indicated. Also shown are

$610 \mathrm{hES}$ and DE stages. Vinculin is used as internal control.

611 (E) IF analysis of PDX1 expression in D7 PDX1 $1^{\mathrm{EGFP} /+}$ HUES4 cells treated with vehicle (Veh),

612 IWP-L6, TGF- $\beta 1$ and/or R115866 as indicated. 
613 (F) Signal tracks of ATAC-seq from Geusz et al. (2021) and SMAD2, EOMES, FOXA2 and OTX2

614 ChIP-seq data from Tsankov et al. (2015) at the CYP26A1 locus. Dashed lines are used to

615 mark areas with differential chromatin accessibility at the DE and PP1 stages, and TF binding

616 at the ME and/or DE stages. ES: ES cells; DE: definitive endoderm; GT: primitive gut tube;

617 PP1: pancreatic progenitor 1, ME: mesendoderm.

618

619 STAR METHODS

620 CONTACT FOR REAGENT AND RESOURCE SHARING

621 Lead contact

622 Further information and requests for resources and reagents may be directed to, and will be

623 fulfilled by the lead contact, Palle Serup (palle.serup@sund.ku.dk)

624

625

Materials availability

626 This study did not generate new unique reagents.

627

628 Data and code availability

629 Sequencing datasets generated in this paper are available at ArrayExpress: E-MTAB-10715.

630

EXPERIMENTAL MODEL AND SUBJECT DETAILS

\section{Cell lines and culture conditions}

633 The human PSC lines H1 (WA01, WiCell; RRID: CVCL_9771) and PDX1 ${ }^{\text {EGF/+ }}$ HUES4 obtained

634 from our facility (Ameri et al., 2017) were maintained in DEF-CS culture media (Takara

635 Biosciences) following manufacturer's instructions with daily media change and passaged

636 every 3-4 days with TrypLE Express Enzyme (Thermo Fisher). All cells were cultured in a

637 humidified $37^{\circ} \mathrm{C}, 5 \% \mathrm{CO}_{2}$ incubator.

638

\section{METHOD DETAILS}

640 Differentiation of hESCs in chemically defined conditions

641 The hESCs were differentiated to pancreatic progenitor cells by a previously described

642 protocol (Rezania et al., 2014) with minor modifications. 150.000 cells/cm2 were seeded in

643 6- or 24-well multi-well plates or in 8-well Ibidi chamber slides after single cell suspension

644 for 3-5 min at $37^{\circ} \mathrm{C}$ in TrypLE Express Enzyme (Thermo Fisher). After $48 \mathrm{~h}$, at day 0 ( 90\% 
confluency), the cells were washed once in $1 \times$ DPBS without $\mathrm{Mg}^{2+}$ and $\mathrm{Ca}^{2+}$ (Thermo Fisher) before addition of S1 basal media (MCDB131, Thermo Fisher) supplemented with Sodium

647 Bicarbonate (1.5g/l, Sigma-Aldrich), Glucose (10mM, Sigma-Aldrich), GlutaMax (1x, Thermo 648 Fisher), BSA (0,5\%, Proliant Biologicals), CHIR-99021 ( $3 \mu \mathrm{M}$, Axon Medchem) and Activin A 649 (100 ng/ml, Peprotech). S1 cells (D3) were washed once in DPBS without $\mathrm{Mg}^{2+}$ and $\mathrm{Ca}^{2+}$ and 650 further supplemented for $2 \mathrm{~d}$ with S2 media including Sodium Bicarbonate $(1.5 \mathrm{~g} / \mathrm{l})$, Glucose 651 (10mM), GlutaMax (1x), BSA (0,5\%), L-Ascorbic Acid (0.25mM, Sigma-Aldrich) and 652 recombinant human FGF7 (KGF) (50ng/ml, Peprotech). During S3-S4, the media was 653 supplemented with Sodium Bicarbonate (2.5g/l), Glucose (10mM), GlutaMax (1x), BSA (2\%), 654 L-Ascorbic Acid (0.25mM), KGF (S3 50ng/ml and S4 2ng/ml), RA (S3 $1 \mu \mathrm{M}$ and S4 0.1 $\mu \mathrm{M}$, 655 Sigma-Aldrich), SANT-1 (0,25 $\mu$ M, Sigma-Aldrich), TPB (S3 200nM and S4 100nM, Sigma656 Aldrich), LDN-193189 (S3 100nM and S4 200nM, Stemgent) and ITS-X (1:200, Thermo 657 Scientific). During S5, the media was supplemented with Sodium Bicarbonate (1.5g/l),

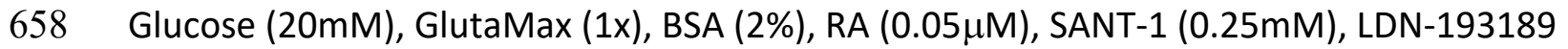

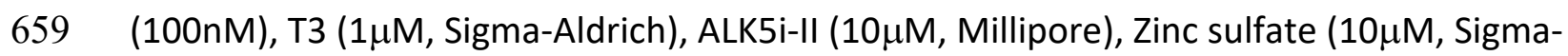
660 Aldrich), Heparin (10 $\mu \mathrm{M}$, Sigma-Aldrich) and ITS-X (1:200). In the initial screen, the media was further supplemented with the following factors during S2-4; Activin A (100ng/ml), TGF-

$662 \beta 1$ (10ng/ml, Peprotech), SB505124 (10 $\mu \mathrm{M}$, Sigma-Aldrich), DAPT (10 $\mu \mathrm{M}$, Selleckchem),

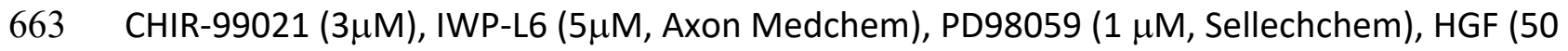
$\mathrm{ng} / \mathrm{ml}$, Peprotech), EGF-L7 (50ng/ml, Peprotech), Wnt3a (100 ng/ml, RnD Systems) or EGF (50ng/ml, Peprotech). In the following experiments, IWP-L6 (5 $\mu \mathrm{M})$ and/or TGF- $\beta 1$ (10ng/ml) were added during S2-3 unless otherwise indicated. When applicable, SB431542 (10 M, Sigma-Aldrich), XAV939 (1 M, Sigma-Aldrich), R115866 (10 M, Sigma-Aldrich), BMP4/6 (50ng/ml, Peprotech), Wnt3a (100ng/ml) or RSPO3 (100ng/ml, Peprotech) were added during S2-3 according to experimental setup.

\section{Flow cytometry}

PDX1 ${ }^{\mathrm{EGFP} /+}$ HUES4 hESCs were differentiated S1-S4 and dissociated at D13 with TrypLE Express Enzyme and washed twice in DPBS without $\mathrm{Mg}^{2+}$ and $\mathrm{Ca}^{2+}+2 \% \mathrm{BSA}$. Resuspended cells were further dissociated in tubes with cell strainer caps (Fisher Scientific), stained with 
DAPI and analyzed on an LSR Fortessa flow cytometer. FACS gating was determined using undifferentiated cells and non-GFP expressing cells.

679 Cells destined for immunofluorescence and confocal microscopy were grown on 24-well

680 plates or 8-well Ibidi $\mu$-slides (Ibidi), respectively. The cells were washed once in DPBS and 681 fixed in $4 \%$ Formaldehyde (VWR) for 30 minutes, then permeabilized using $0.5 \%$ Triton-X in

682 DPBS for $10 \mathrm{~min}$ at room temperature and blocked in SuperBlock (Thermo Fisher) for 30 683 minutes.

684 Primary antibodies (see Key Resources Table) were diluted in 0.1\% Triton X-100 in DPBS. 685 Incubation was done at $4^{\circ} \mathrm{C}$ overnight followed by $3 \times 5$ minutes wash in DPBS. Secondary 686 antibodies (1:500, raised in Donkey) conjugated to either Alexa Fluor 488, Cy3 or Cy5 (all 687 Jackson ImmunoResearch) were incubated for 45 minutes at room temperature and then 688 washed $3 \times 5 \mathrm{~min}$ in DPBS followed by nuclear staining with DAPI (Thermo Fisher). FZD receptor expression was detected using a human Fc-tagged version of the NGS Wnt ligand: DRPB-Fz7/8 (Miao et al., 2020). Control and TGF- $\beta 1$ treated cells, grown on 8-well Ibidi $\mu$ slides (Ibidi), were fixed, permeabilized and incubated for $1 \mathrm{~h}$ in media conditioned with cells expressing DRPB-Fz7/8 together with a primary antibody against $\beta$-catenin (BD Biosciences).

693 Detection of DRPB-Fz7/8 was done with a PE-tagged anti-human Fc antibody (Jackson

694 ImmunoResearch).

695 Images were captured and processed on a Zeiss Axioobserver using Plan-Apochromat $69610 x / 0.45$ and Plan-Apochromat 20x/0.8 objectives and ZEN software. Confocal images were 697 captured on a Zeiss LSM780 confocal microscope using a Plan-Apochromat 63x/1.40 Oil 698 objective. Figures were prepared using Adobe Photoshop CS6 and Adobe Illustrator CS6 699 (Adobe Systems, San Jose, CA, USA). RNA extraction and quantitative real-time PCR Total RNA was extracted with the RNeasy Plus Mini kit (Qiagen) and reverse-transcribed using the SuperScript III First-Strand synthesis kit (Invitrogen/Thermo Fisher). Quantitative real-time PCR experiments were performed using the StepOnePLus system (Applied 
Table S7 for primers. Relative changes in gene expression was compared to undifferentiated hESCs using the $\Delta \Delta C$ t method.

\section{Western blotting}

710 Harvested cells were lysed in RIPA buffer containing 1x phosphatase inhibitor cocktail

711 (Sigma-Aldrich) and cOmplete Ultra Protease inhibitor (Thermo Scientific/Roche) on ice for

$71210 \mathrm{~min}$. Cell lysates were sonicated $5 \times 30 \mathrm{sec}$ ON/OFF on a Diagenode BioRuptor in $1.5 \mathrm{~mL}$

713 eppendorff tubes followed by centrifugation at $21000 \mathrm{~g}$ for 30 minutes at $4^{\circ} \mathrm{C}$ and saving the

714 supernatant. Pierce BCA protein kit (ThermoFisher) was used to measure protein concentration on a Nanodrop 2000 (ThermoFisher). Lysates were boiled for 5 minutes in

716 Laemmli sample buffer and 20-40 $\mu \mathrm{g}$ protein was separated by electrophoresis on NuPage

$7174-12 \%$ BisTris SDS-PAGE gels in MOPS buffer (Thermo Fisher) and transferred to PVDF membranes (Bio-Rad) using the BioRad Mini-Protean transfer system. Membranes were

719 blocked in SuperBlock (Thermo Fisher) for 1 hour at room temperature and incubated with primary antibodies (see Key Resources Table) overnight at $4{ }^{\circ} \mathrm{C}$. After three washes with TBS-T (0.1\% Tween-20 in 1x Tris-buffered saline), the blot was incubated with respective secondary HRP antibodies at room temperature for 30 minutes. ECL Prime Western Blotting Detection Reagent was used for detection according to the manufacturer's instruction

724 (Sigma-Aldrich). For re-blotting, antibodies were stripped by Restore Western Blot Stripping Buffer (Thermo Fisher).

\section{EdU-incorporation, analysis and quantification}

$728 P D X 1^{\mathrm{EGFP} /+}$ HUES4 cells were labeled with EdU and detected according to the Click-iT EdU

729 Alexa Fluor 594 Flow Cytometry Assay kit (Thermo Fisher) protocol at D5 and D7 of

730 differentiation. In brief, 10 M EdU was added to the differentiation culture medium and

731 cells were harvested and dissociated in to a single-cell suspension after $3 \mathrm{~h}$. The cells were

732 fixed and permeabilized followed by EdU detection with the Click-iT EdU reagent and

733 nuclear staining with DAPI. FACS gating was determined using non-EdU treated cells and

734 EdU-treated cells without detection reagent. Cells were analyzed using a LSR Fortessa flow

735 cytometer. Alternatively, EdU-treated cells were fixed, permeabilized and stained according

736 to the Click-iT Plus EdU Cell Proliferation Kit for Imaging, 647 dye (ThermoFisher). 
$\mathrm{OTX}^{+}$and $\mathrm{OTX} 2^{+} \mathrm{EdU}^{+}$cells were quantified from confocal images acquired on an LSM780 confocal microscope as described above. Cells were identified using the Spot function in Imaris $^{\mathrm{TM}}$ (Bitplane) with the diameter set to $4.15 \mu \mathrm{m}$ and the Co-localization function with the maximum distance between spots set to $\leq 2 \mu \mathrm{m}$ to identify co-expressing cells.

\section{RNA-seq}

743 RNA-seq libraries were built using biological triplicates from PDX1 ${ }^{\text {EGFP/+ }}$ HUES4 cells and 744 duplicates from $\mathrm{H} 1$ cells with $1 \mu \mathrm{g}$ of RNA using the NEB NEXT Ultra II RNA Library Prep Kit 745 (NEB \#E7770) and mRNA magnetic isolation module for poly(A) purification (NEB \#E7490) with 5 cycles of amplification. Quality of the RNA and subsequently of the libraries were measured on a Fragment Analyser and the libraries were loaded accordingly and barcoded with NEB Next multiplex Oligos for Illumina (E7335 and E7500) on Illumina NextSeq 500 with Hi-output $1 \times 75 b p$ kit. FASTQ-files were generated using bcl2Fastq (Illumina) and aligned to hg38 human genome using STAR (Dobin et al., 2013) with standard alignment settings resulting in >90\% alignment of reads. Quantified gene count matrix with STAR (flag: --quantMode GeneCounts) and loaded into R using DESEQ2 package for differential expression analysis on gene level (Love et al., 2014). We performed quality control assessment including Principal Component Analysis after regularized logarithmic transformation. For the differential expression analysis for each cell line on each day we tested control (ctrl) vs. IWP-L6, TGF- $\beta$ and IWP-L6+TGF- $\beta$ $(I+T)$. From these comparisons, scaled counts for all deregulated genes with log2FoldChange $>1$ and adjusted $p$-value $<0.05$ are shown in Figure 2 to provide an overview of effects and heterogeneity. Clusters were obtained with kmeans clustering using the superheat package. Gene lengths were obtained with EDAseq package used to provide gene length normalised read counts (RPKM) for supplementary files (Risso et al., 2011). Used ggplot for plotting except for heatmaps. Code for differential expression analysis available upon request.

764 Gene Set Enrichment Analysis (GSEA) was done using a GSEA software downloaded from the Broad Institute (Mootha et al., 2003; Subramanian et al., 2005). DESeq2 results were imported based on Wald Statistics as preranked lists and enrichment was calculated with the classic setting and 1000 permutations. Gene sets were downloaded from the Molecular Signature Database; mSigDB v7.2 (Chang et al., 2004; Hsiao et al., 2001; Liberzon et al., 
2011). We performed GSEA against the entire C5: ontology gene sets (Table S3) and C8: cell type signature gene sets (Table S5) from mSigDB v7.2 as well as against selected gene sets representing cycling genes, liver-specific genes and genes enriched in developing human pancreas and liver. RNA-seq data generated in this study are available at the ArrayExpress database under the accession number E-MTAB-10715.

\section{Public datasets}

776 The ATAC-seq data (GSE149148, Geusz et al., 2021) and the ChIP-seq data (GSE61475,

777 Tsankov et al., 2015) used in this study were downloaded from Gene Expression Omnibus

778 (GEO) as processed data in bigwig and bed formats.

\section{Statistical analysis and reproducibility}

781 All statistics were performed using GraphPad Prism 8 software (GraphPad). Data sets with two groups having equal variances were analyzed by a two-tailed Student's $t$ test. For data with unequal variances, two-tailed Welch's t tests were applied. Comparison of three or more groups was performed by one-way analysis of variance (ANOVA) followed by either Dunnett's test using ctrl samples as reference, or Tukey's test for comparison of all means. Statistics for differential expression of RNA-seq are provided as padj values from the DESeq2 analysis. $P$ values are displayed in the figures and sample sizes are provided in the figure legends. Statistical significance is defined as $p<0.05$ for qRT-PCR and GSEA data as well as FACS and IF image quantifications, while the DESeq2 analysis used an adjusted p-value (padj) cut-off set to 0.1 (default). 
791

792

793

794

795

796

797

798

799

800

801

802

803

804

805

806

807

808

809

810

811

812

813

814

815

816

817

818

819

820

821

822

823

824

825

826

827

828

829

830

831

832

833

834

835

836

\section{References}

Abu-Abed, S., Dolle, P., Metzger, D., Beckett, B., Chambon, P., and Petkovich, M. (2001). The retinoic acid-metabolizing enzyme, CYP26A1, is essential for normal hindbrain patterning, vertebral identity, and development of posterior structures. Genes Dev 15, 226-240.

Abu-Abed, S., Dolle, P., Metzger, D., Wood, C., MacLean, G., Chambon, P., and Petkovich, M. (2003). Developing with lethal RA levels: genetic ablation of Rarg can restore the viability of mice lacking Cyp26a1. Development 130, 1449-1459.

Ameri, J., Borup, R., Prawiro, C., Ramond, C., Schachter, K.A., Scharfmann, R., and Semb, H. (2017). Efficient Generation of Glucose-Responsive Beta Cells from Isolated GP2(+) Human Pancreatic Progenitors. Cell Rep 19, 36-49.

Arnold, S.J., and Robertson, E.J. (2009). Making a commitment: cell lineage allocation and axis patterning in the early mouse embryo. Nat Rev Mol Cell Biol 10, 91-103.

Bayha, E., Jorgensen, M.C., Serup, P., and Grapin-Botton, A. (2009). Retinoic Acid Signaling Organizes Endodermal Organ Specification along the Entire Antero-Posterior Axis. Plos One 4.

Belo, J.A., Bachiller, D., Agius, E., Kemp, C., Borges, A.C., Marques, S., Piccolo, S., and De Robertis, E.M. (2000). Cerberus-like is a secreted BMP and nodal antagonist not essential for mouse development. Genesis 26, 265-270.

Bhushan, A., Itoh, N., Kato, S., Thiery, J.P., Czernichow, P., Bellusci, S., and Scharfmann, R. (2001). Fgf10 is essential for maintaining the proliferative capacity of epithelial progenitor cells during early pancreatic organogenesis. Development 128, 5109-5117.

Bunt, J., Hasselt, N.E., Zwijnenburg, D.A., Hamdi, M., Koster, J., Versteeg, R., and Kool, M. (2012). OTX2 directly activates cell cycle genes and inhibits differentiation in medulloblastoma cells. Int J Cancer 131, E21-32.

Candia, A.F., Watabe, T., Hawley, S.H., Onichtchouk, D., Zhang, Y., Derynck, R., Niehrs, C., and Cho, K.W. (1997). Cellular interpretation of multiple TGF-beta signals: intracellular antagonism between activin/BVg1 and BMP-2/4 signaling mediated by Smads. Development 124, 4467-4480.

Chang, H.Y., Sneddon, J.B., Alizadeh, A.A., Sood, R., West, R.B., Montgomery, K., Chi, J.T., van de Rijn, M., Botstein, D., and Brown, P.O. (2004). Gene expression signature of fibroblast serum response predicts human cancer progression: similarities between tumors and wounds. PLoS Biol 2, E7.

Chung, W.S., Shin, C.H., and Stainier, D.Y. (2008). Bmp2 signaling regulates the hepatic versus pancreatic fate decision. Dev Cell 15, 738-748.

Costello, I., Nowotschin, S., Sun, X., Mould, A.W., Hadjantonakis, A.K., Bikoff, E.K., and Robertson, E.J. (2015). Lhx1 functions together with Otx2, Foxa2, and Ldb1 to govern anterior mesendoderm, node, and midline development. Genes Dev 29, 2108-2122.

Dang, L.T., Miao, Y., Ha, A., Yuki, K., Park, K., Janda, C.Y., Jude, K.M., Mohan, K., Ha, N., Vallon, M., et al. (2019). Receptor subtype discrimination using extensive shape complementary designed interfaces. Nat Struct Mol Biol 26, 407-414.

Deimling, S.J., and Drysdale, T.A. (2009). Retinoic acid regulates anterior-posterior patterning within the lateral plate mesoderm of Xenopus. Mech Dev 126, 913-923. Deutsch, G., Jung, J., Zheng, M., Lora, J., and Zaret, K.S. (2001). A bipotential precursor population for pancreas and liver within the embryonic endoderm. Development 128, 871881. 
Dobin, A., Davis, C.A., Schlesinger, F., Drenkow, J., Zaleski, C., Jha, S., Batut, P., Chaisson, M., and Gingeras, T.R. (2013). STAR: ultrafast universal RNA-seq aligner. Bioinformatics 29, 1521.

840 Fossat, N., Ip, C.K., Jones, V.J., Studdert, J.B., Khoo, P.L., Lewis, S.L., Power, M., Tourle, K., 841 Loebel, D.A., Kwan, K.M., et al. (2015). Context-specific function of the LIM homeobox 1 transcription factor in head formation of the mouse embryo. Development 142, 2069-2079. Galvin, K.E., Travis, E.D., Yee, D., Magnuson, T., and Vivian, J.L. (2010). Nodal signaling regulates the bone morphogenic protein pluripotency pathway in mouse embryonic stem cells. J Biol Chem 285, 19747-19756. Gestri, G., Carl, M., Appolloni, I., Wilson, S.W., Barsacchi, G., and Andreazzoli, M. (2005). Six3 functions in anterior neural plate specification by promoting cell proliferation and inhibiting Bmp4 expression. Development 132, 2401-2413. Geusz, R.J., Wang, A., Chiou, J., Lancman, J.J., Wetton, N., Kefalopoulou, S., Wang, J., Qiu, Y., Yan, J., Aylward, A., et al. (2021). Pancreatic progenitor epigenome maps prioritize type 2 diabetes risk genes with roles in development. Elife 10. Goss, A.M., Tian, Y., Tsukiyama, T., Cohen, E.D., Zhou, D., Lu, M.M., Yamaguchi, T.P., and Morrisey, E.E. (2009). Wnt2/2b and beta-Catenin Signaling Are Necessary and Sufficient to Specify Lung Progenitors in the Foregut. Developmental Cell 17, 290-298. Grapin-Botton, A. (2005). Antero-posterior patterning of the vertebrate digestive tract: 40 years after Nicole Le Douarin's PhD thesis. International Journal of Developmental Biology 49, 335-347.

858 Hsiao, L.L., Dangond, F., Yoshida, T., Hong, R., Jensen, R.V., Misra, J., Dillon, W., Lee, K.F., 859 Clark, K.E., Haverty, P., et al. (2001). A compendium of gene expression in normal human tissues. Physiol Genomics 7, 97-104.

861 Ip, C.K., Fossat, N., Jones, V., Lamonerie, T., and Tam, P.P. (2014). Head formation: OTX2 regulates Dkk1 and Lhx1 activity in the anterior mesendoderm. Development 141, 38593867. Jennings, R.E., Berry, A.A., Gerrard, D.T., Wearne, S.J., Strutt, J., Withey, S., Chhatriwala, M., Piper Hanley, K., Vallier, L., Bobola, N., et al. (2017). Laser Capture and Deep Sequencing Reveals the Transcriptomic Programmes Regulating the Onset of Pancreas and Liver Differentiation in Human Embryos. Stem Cell Reports 9, 1387-1394. Jung, J., Zheng, M., Goldfarb, M., and Zaret, K.S. (1999). Initiation of mammalian liver development from endoderm by fibroblast growth factors. Science 284, 1998-2003. Kraus, M.R.C., and Grapin-Botton, A. (2012). Patterning and shaping the endoderm in vivo and in culture. Current Opinion in Genetics \& Development 22, 347-353. Lagutin, O.V., Zhu, C.C., Kobayashi, D., Topczewski, J., Shimamura, K., Puelles, L., Russell, H.R., McKinnon, P.J., Solnica-Krezel, L., and Oliver, G. (2003). Six3 repression of Wnt signaling in the anterior neuroectoderm is essential for vertebrate forebrain development. Genes Dev 17, 368-379.

876 Li, Y., Rankin, S.A., Sinner, D., Kenny, A.P., Krieg, P.A., and Zorn, A.M. (2008). Sfrp5 877 coordinates foregut specification and morphogenesis by antagonizing both canonical and 878 noncanonical Wnt11 signaling. Genes Dev 22, 3050-3063.

879 Liberzon, A., Subramanian, A., Pinchback, R., Thorvaldsdottir, H., Tamayo, P., and Mesirov, 880 J.P. (2011). Molecular signatures database (MSigDB) 3.0. Bioinformatics 27, 1739-1740. 881 Loh, K.M., Ang, L.T., Zhang, J., Kumar, V., Ang, J., Auyeong, J.Q., Lee, K.L., Choo, S.H., Lim, 882 C.Y., Nichane, M., et al. (2014). Efficient endoderm induction from human pluripotent stem 883 cells by logically directing signals controlling lineage bifurcations. Cell Stem Cell 14, 237-252. 
884

885

886

887

888

889

890

891

892

893

894

895

896

897

898

899

900

901

902

903

904

905

906

907

908

909

910

911

912

913

914

915

916

917

918

919

920

921

922

923

924

925

926

927

928

929

Love, M.I., Huber, W., and Anders, S. (2014). Moderated estimation of fold change and dispersion for RNA-seq data with DESeq2. Genome Biol 15, 550.

Mahaddalkar, P.U., Scheibner, K., Pfluger, S., Ansarullah, Sterr, M., Beckenbauer, J., Irmler, M., Beckers, J., Knobel, S., and Lickert, H. (2020). Generation of pancreatic beta cells from CD177(+) anterior definitive endoderm. Nat Biotechnol 38, 1061-1072.

McLin, V.A., Rankin, S.A., and Zorn, A.M. (2007). Repression of Wnt/beta-catenin signaling in the anterior endoderm is essential for liver and pancreas development. Development 134, 2207-2217.

McMahon, J.A., and McMahon, A.P. (1989). Nucleotide sequence, chromosomal localization and developmental expression of the mouse int-1-related gene. Development 107, 643-650. Miao, Y., Ha, A., de Lau, W., Yuki, K., Santos, A.J.M., You, C., Geurts, M.H., Puschhof, J., Pleguezuelos-Manzano, C., Peng, W.C., et al. (2020). Next-Generation Surrogate Wnts Support Organoid Growth and Deconvolute Frizzled Pleiotropy In Vivo. Cell Stem Cell 27, 840-851 e846.

Micallef, S.J., Janes, M.E., Knezevic, K., Davis, R.P., Elefanty, A.G., and Stanley, E.G. (2005). Retinoic acid induces Pdx1-positive endoderm in differentiating mouse embryonic stem cells. Diabetes 54, 301-305.

Mikels, A.J., and Nusse, R. (2006). Purified Wnt5a protein activates or inhibits beta-cateninTCF signaling depending on receptor context. PLoS Biol 4, e115.

Molotkov, A., Molotkova, N., and Duester, G. (2005). Retinoic acid generated by Raldh2 in mesoderm is required for mouse dorsal Endodermal pancreas development. Developmental Dynamics 232, 950-957.

Monkley, S.J., Delaney, S.J., Pennisi, D.J., Christiansen, J.H., and Wainwright, B.J. (1996). Targeted disruption of the Wnt2 gene results in placentation defects. Development 122, 3343-3353.

Mootha, V.K., Lindgren, C.M., Eriksson, K.F., Subramanian, A., Sihag, S., Lehar, J., Puigserver, P., Carlsson, E., Ridderstrale, M., Laurila, E., et al. (2003). PGC-1alpha-responsive genes involved in oxidative phosphorylation are coordinately downregulated in human diabetes. Nat Genet 34, 267-273.

Nostro, M.C., Sarangi, F., Ogawa, S., Holtzinger, A., Corneo, B., Li, X., Micallef, S.J., Park, I.H., Basford, C., Wheeler, M.B., et al. (2011). Stage-specific signaling through TGFbeta family members and WNT regulates patterning and pancreatic specification of human pluripotent stem cells. Development 138, 861-871.

Ober, E.A., Verkade, H., Field, H.A., and Stainier, D.Y. (2006). Mesodermal Wnt2b signalling positively regulates liver specification. Nature 442, 688-691.

Onishi, K., and Zou, Y.M. (2017). Sonic Hedgehog switches on Wnt/planar cell polarity signaling in commissural axon growth cones by reducing levels of Shisa2. Elife 6.

Ortmann, D., Brown, S., Czechanski, A., Aydin, S., Muraro, D., Huang, Y., Tomaz, R.A., Osnato, A., Canu, G., Wesley, B.T., et al. (2020). Naive Pluripotent Stem Cells Exhibit Phenotypic Variability that Is Driven by Genetic Variation. Cell Stem Cell 27, 470-481 e476. Pagliuca, F.W., Millman, J.R., Gurtler, M., Segel, M., Van Dervort, A., Ryu, J.H., Peterson, Q.P., Greiner, D., and Melton, D.A. (2014). Generation of functional human pancreatic beta cells in vitro. Cell 159, 428-439.

Piccolo, S., Agius, E., Leyns, L., Bhattacharyya, S., Grunz, H., Bouwmeester, T., and De Robertis, E.M. (1999). The head inducer Cerberus is a multifunctional antagonist of Nodal, BMP and Wnt signals. Nature 397, 707-710. 
930

931

932

933

934

935

936

937

938

939

940

941

942

943

944

945

946

947

948

949

950

951

952

953

954

955

956

957

958

959

960

961

962

963

964

965

966

967

968

969

970

971

972

973

974

975

976

Poulain, M., and Ober, E.A. (2011). Interplay between Wnt2 and Wnt2bb controls multiple steps of early foregut-derived organ development. Development 138, 3557-3568.

Rankin, S.A., Kormish, J., Kofron, M., Jegga, A., and Zorn, A.M. (2011). A gene regulatory network controlling hhex transcription in the anterior endoderm of the organizer. Dev Biol 351, 297-310.

Rankin, S.A., McCracken, K.W., Luedeke, D.M., Han, L., Wells, J.M., Shannon, J.M., and Zorn, A.M. (2018). Timing is everything: Reiterative Wnt, BMP and RA signaling regulate developmental competence during endoderm organogenesis. Dev Biol 434, 121-132. Rezania, A., Bruin, J.E., Arora, P., Rubin, A., Batushansky, I., Asadi, A., O'Dwyer, S., Quiskamp, N., Mojibian, M., Albrecht, T., et al. (2014). Reversal of diabetes with insulin-producing cells derived in vitro from human pluripotent stem cells. Nat Biotechnol 32, 1121-1133.

Rezania, A., Riedel, M.J., Wideman, R.D., Karanu, F., Ao, Z., Warnock, G.L., and Kieffer, T.J. (2011). Production of functional glucagon-secreting alpha-cells from human embryonic stem cells. Diabetes 60, 239-247.

Ribes, V., Fraulob, V., Petkovich, M., and Dolle, P. (2007). The oxidizing enzyme CYP26a1 tightly regulates the availability of retinoic acid in the gastrulating mouse embryo to ensure proper head development and vasculogenesis. Dev Dyn 236, 644-653.

Risso, D., Schwartz, K., Sherlock, G., and Dudoit, S. (2011). GC-content normalization for RNA-Seq data. BMC Bioinformatics 12, 480.

Rodriguez-Seguel, E., Mah, N., Naumann, H., Pongrac, I.M., Cerda-Esteban, N., Fontaine, J.F., Wang, Y., Chen, W., Andrade-Navarro, M.A., and Spagnoli, F.M. (2013). Mutually exclusive signaling signatures define the hepatic and pancreatic progenitor cell lineage divergence. Genes Dev 27, 1932-1946.

Rossi, J.M., Dunn, N.R., Hogan, B.L.M., and Zaret, K.S. (2001). Distinct mesodermal signals, including BMPs from the septum transversum mesenchyme, are required in combination for hepatogenesis from the endoderm. Genes \& Development 15, 1998-2009.

Sanvitale, C.E., Kerr, G., Chaikuad, A., Ramel, M.C., Mohedas, A.H., Reichert, S., Wang, Y., Triffitt, J.T., Cuny, G.D., Yu, P.B., et al. (2013). A new class of small molecule inhibitor of BMP signaling. PLoS One 8, e62721.

Seiliez, I., Thisse, B., and Thisse, C. (2006). FoxA3 and goosecoid promote anterior neural fate through inhibition of Wnt8a activity before the onset of gastrulation. Dev Biol 290, 152163.

Serls, A.E., Doherty, S., Parvatiyar, P., Wells, J.M., and Deutsch, G.H. (2005). Different thresholds of fibroblast growth factors pattern the ventral foregut into liver and lung. Development 132, 35-47.

Shim, W.J., Sinniah, E., Xu, J., Vitrinel, B., Alexanian, M., Andreoletti, G., Shen, S., Sun, Y., Balderson, B., Boix, C., et al. (2020). Conserved Epigenetic Regulatory Logic Infers Genes Governing Cell Identity. Cell Syst 11, 625-639 e613.

Sibbritt, T., Ip, C.K., Khoo, P.L., Wilkie, E., Jones, V., Sun, J.Q.J., Shen, J.X., Peng, G., Han, J.J., Jing, N., et al. (2018). A gene regulatory network anchored by LIM homeobox 1 for embryonic head development. Genesis 56, e23246.

Spence, J.R., Lauf, R., and Shroyer, N.F. (2011). Vertebrate intestinal endoderm development. Dev Dyn 240, 501-520.

Stafford, D., and Prince, V.E. (2002). Retinoic acid signaling is required for a critical early step in zebrafish pancreatic development. Current Biology 12, 1215-1220.

Stevens, M.L., Chaturvedi, P., Rankin, S.A., Macdonald, M., Jagannathan, S., Yukawa, M., Barski, A., and Zorn, A.M. (2017). Genomic integration of Wnt/beta-catenin and 
$\mathrm{BMP} / \mathrm{Smad} 1$ signaling coordinates foregut and hindgut transcriptional programs.

978 Development 144, 1283-1295.

979 Subramanian, A., Tamayo, P., Mootha, V.K., Mukherjee, S., Ebert, B.L., Gillette, M.A.,

980 Paulovich, A., Pomeroy, S.L., Golub, T.R., Lander, E.S., et al. (2005). Gene set enrichment

981 analysis: a knowledge-based approach for interpreting genome-wide expression profiles.

982 Proc Natl Acad Sci U S A 102, 15545-15550.

983 Tam, P.P., and Loebel, D.A. (2007). Gene function in mouse embryogenesis: get set for

984 gastrulation. Nat Rev Genet 8, 368-381.

985 Tao, Q., Yokota, C., Puck, H., Kofron, M., Birsoy, B., Yan, D., Asashima, M., Wylie, C.C., Lin, X., 986 and Heasman, J. (2005). Maternal wnt11 activates the canonical wnt signaling pathway

987 required for axis formation in Xenopus embryos. Cell 120, 857-871.

988 Tsankov, A.M., Gu, H., Akopian, V., Ziller, M.J., Donaghey, J., Amit, I., Gnirke, A., and

989 Meissner, A. (2015). Transcription factor binding dynamics during human ES cell

990 differentiation. Nature 518, 344-349.

991 Wandzioch, E., and Zaret, K.S. (2009). Dynamic signaling network for the specification of

992 embryonic pancreas and liver progenitors. Science 324, 1707-1710.

993 Yamamoto, A., Nagano, T., Takehara, S., Hibi, M., and Aizawa, S. (2005). Shisa promotes

994 head formation through the inhibition of receptor protein maturation for the caudalizing

995 factors, Wnt and FGF. Cell 120, 223-235.

996 Yao, J., and Kessler, D.S. (2001). Goosecoid promotes head organizer activity by direct

997 repression of Xwnt8 in Spemann's organizer. Development 128, 2975-2987.

998 Yasuoka, Y., Suzuki, Y., Takahashi, S., Someya, H., Sudou, N., Haramoto, Y., Cho, K.W.,

999 Asashima, M., Sugano, S., and Taira, M. (2014). Occupancy of tissue-specific cis-regulatory

1000 modules by Otx2 and TLE/Groucho for embryonic head specification. Nat Commun 5, 4322.

1001 Zakin, L.D., Mazan, S., Maury, M., Martin, N., Guenet, J.L., and Brulet, P. (1998). Structure

1002 and expression of Wnt13, a novel mouse Wnt2 related gene. Mech Dev 73, 107-116.

1003 Zaret, K.S. (2008). Genetic programming of liver and pancreas progenitors: lessons for stem-

1004 cell differentiation. Nat Rev Genet 9, 329-340. 
bioRxiv preprint doi: https://doi.org/10.1101/2021.07.15.452587; this version posted July 16, 2021. The copyright holder for this preprint

(which was not certified by peer review) is the author/funder, who has granted bioRxiv a license to display the preprint in perpetuity. It is made available under aCC-BY-NC-ND 4.0 International license.

A

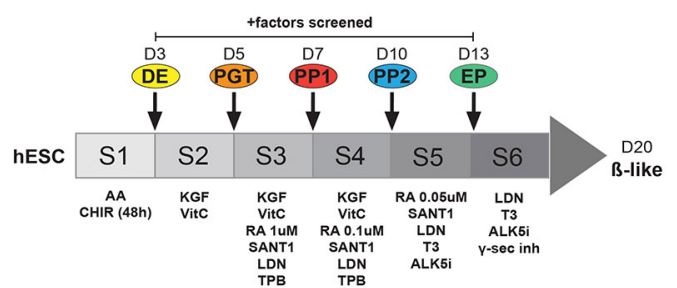

D

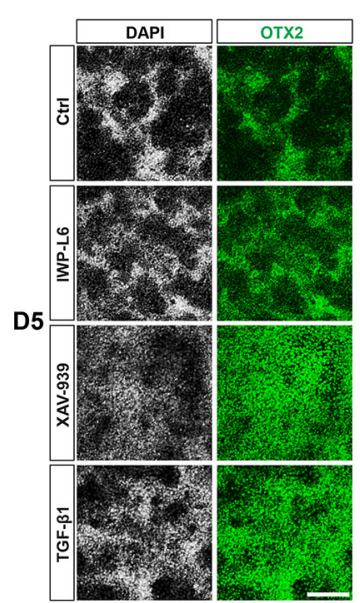

F

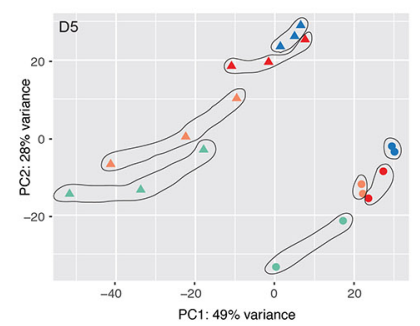

G

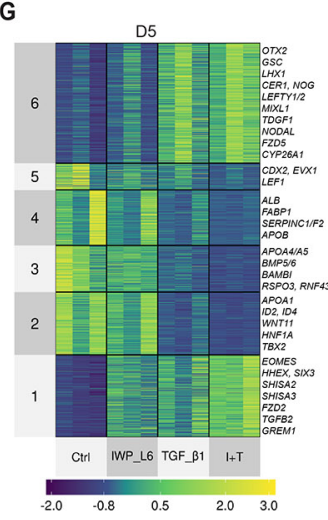

J

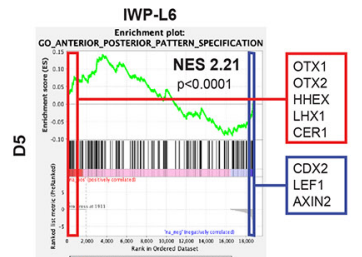

E
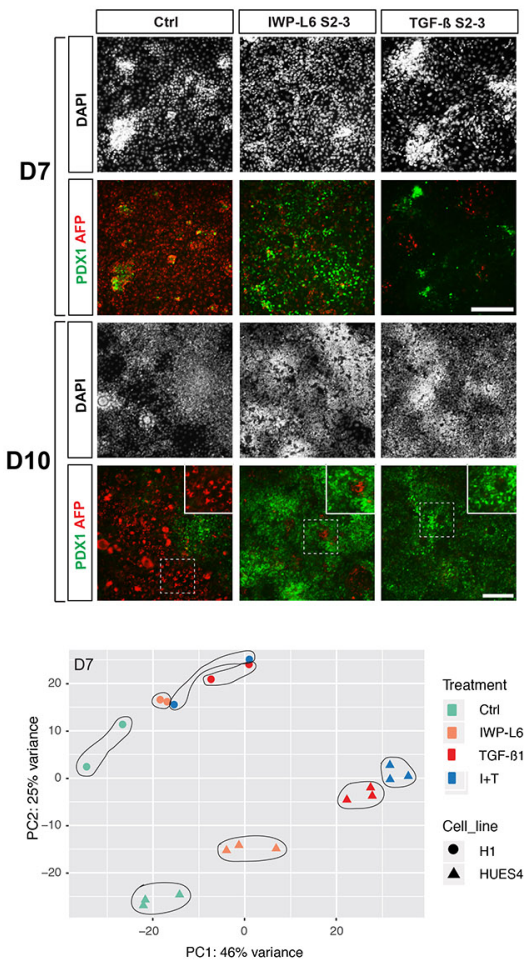

H
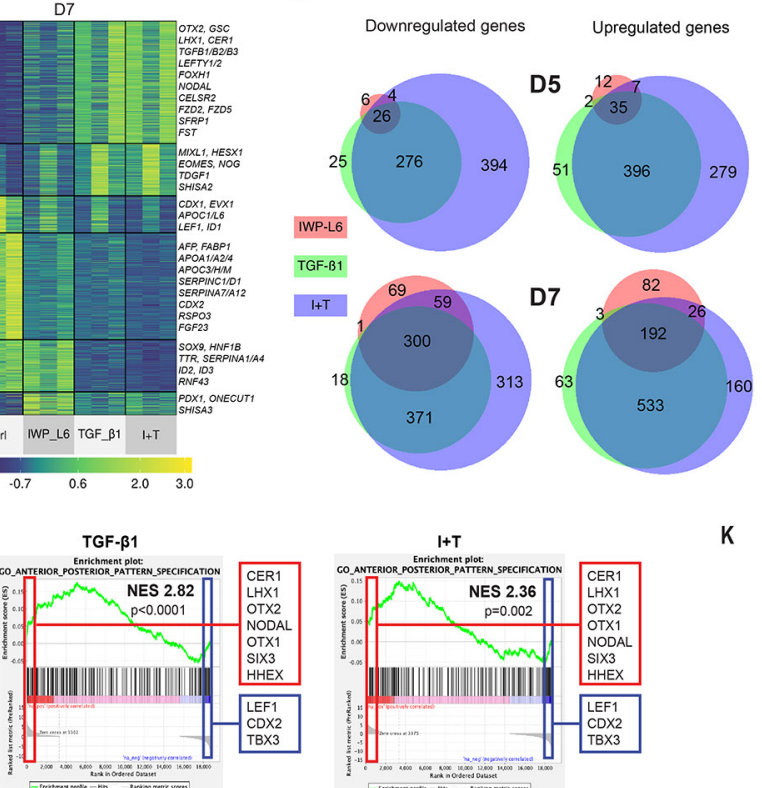

K

$B$

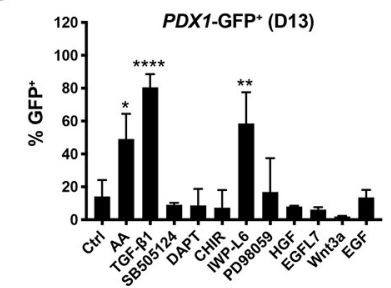

IWP-L6

$1+T$

- H1

HUES4

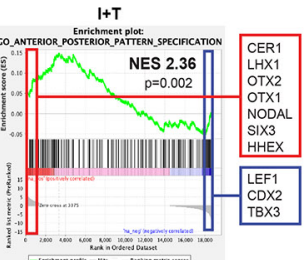

C
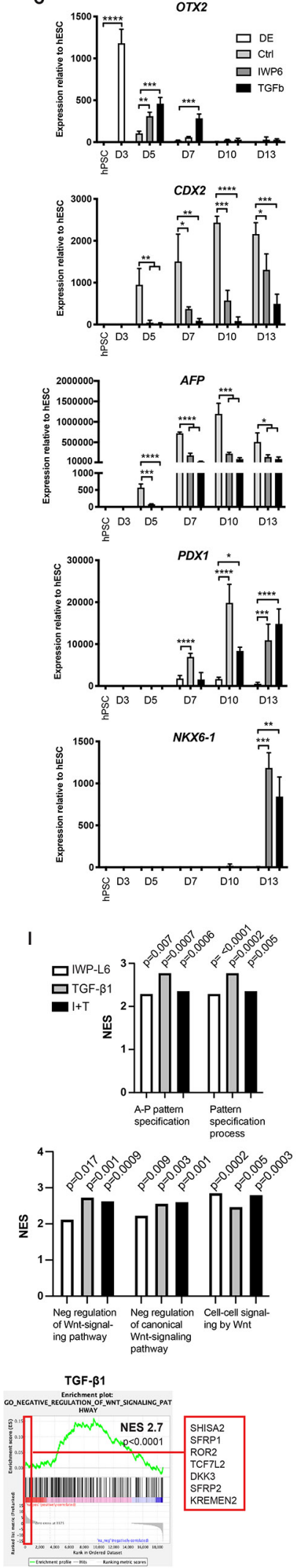

Figure 1 
bioRxiv preprint doi: https://doi.org/10.1101/2021.07.15.452587; this version posted July 16, 2021. The copyright holder for this preprint

(which was not certified by peer review) is the author/funder, who has granted bioRxiv a license to display the preprint in perpetuity. It is made available under aCC-BY-NC-ND 4.0 International license.

A

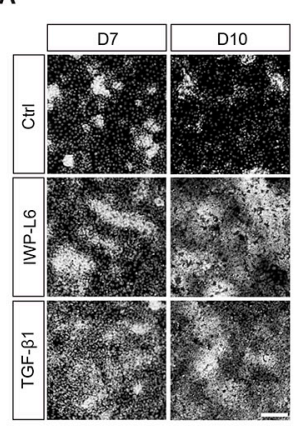

B

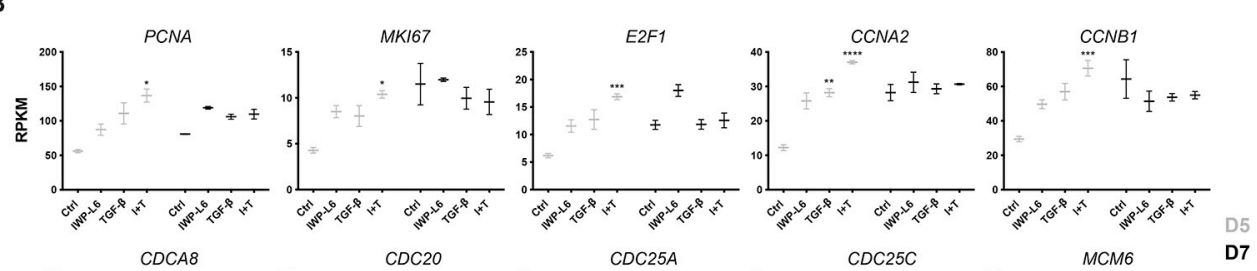

c

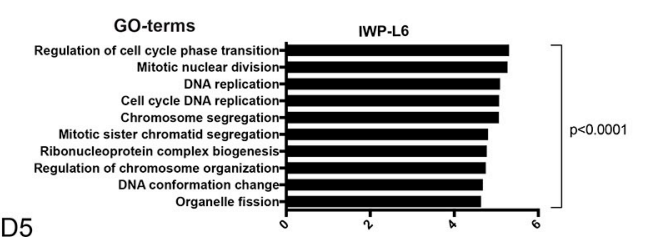

D5

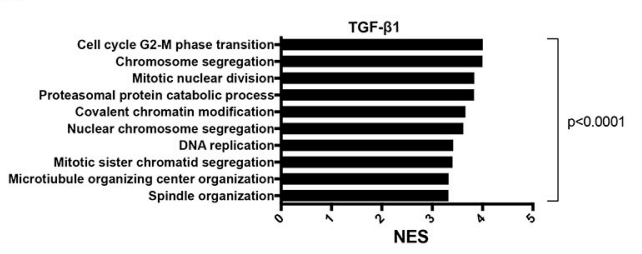

D

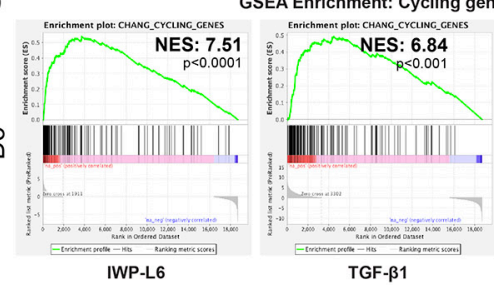

G

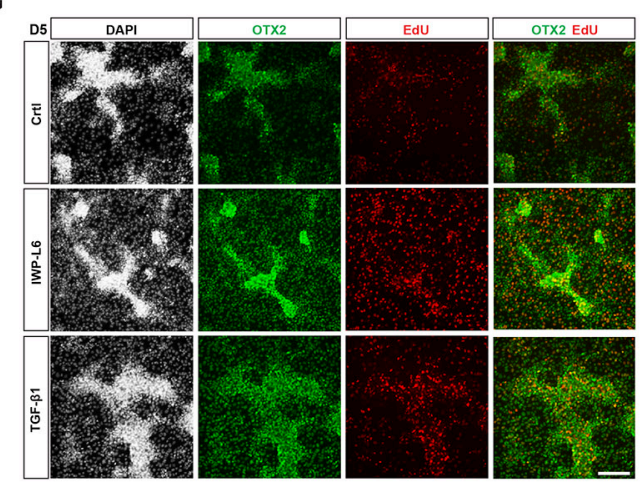

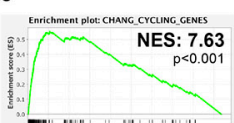

페IIIIIII!

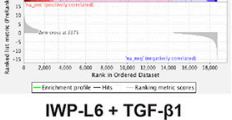

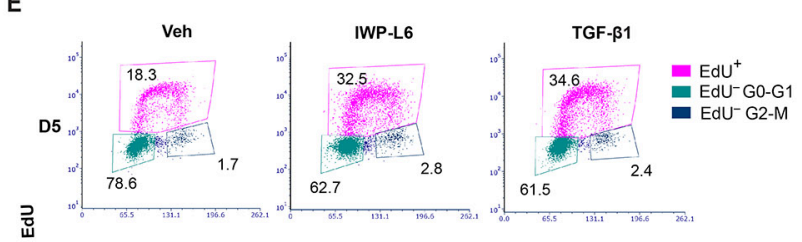

흠

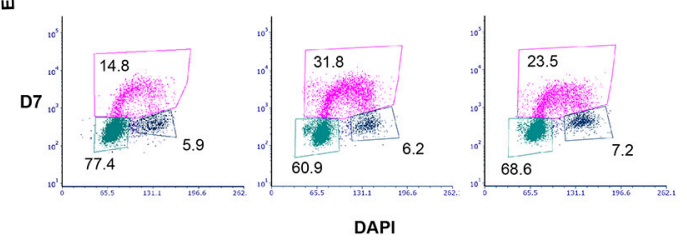

F

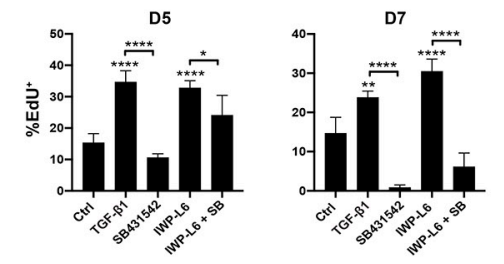

H
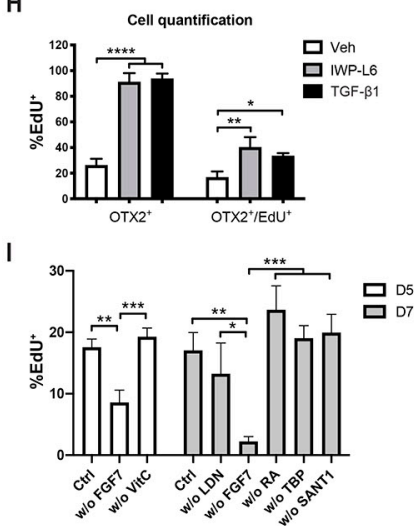

Figure 2
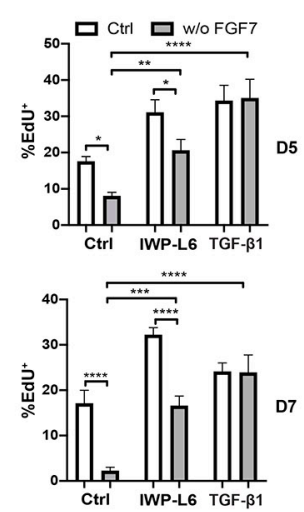
bioRxiv preprint doi: https://doi.org/10.1101/2021.07.15.452587; this version posted July 16, 2021. The copyright holder for this preprint

(which was not certified by peer review) is the author/funder, who has granted bioRxiv a license to display the preprint in perpetuity. It is made available under aCC-BY-NC-ND 4.0 International license.

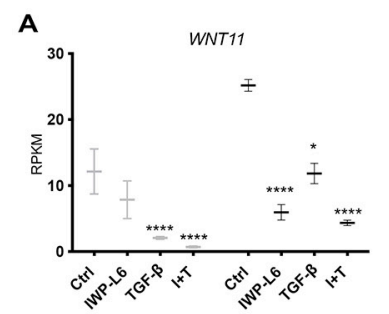

B
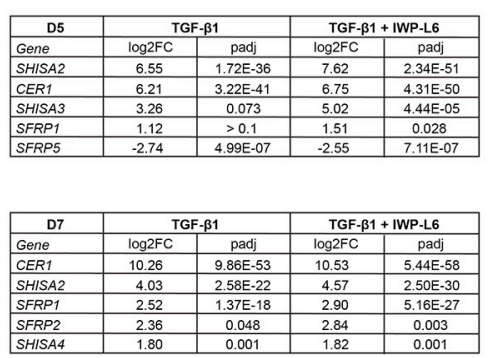

D

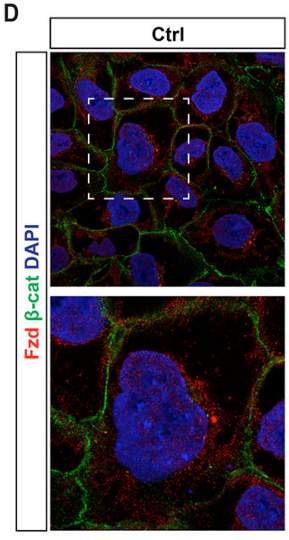

E

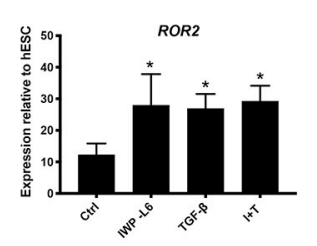

TGF- $\beta 1$

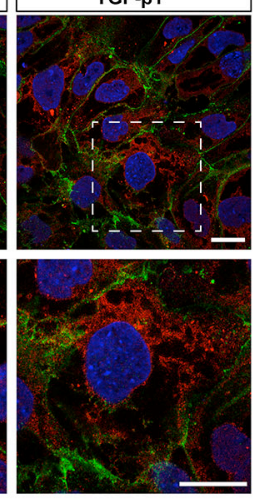

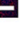

C
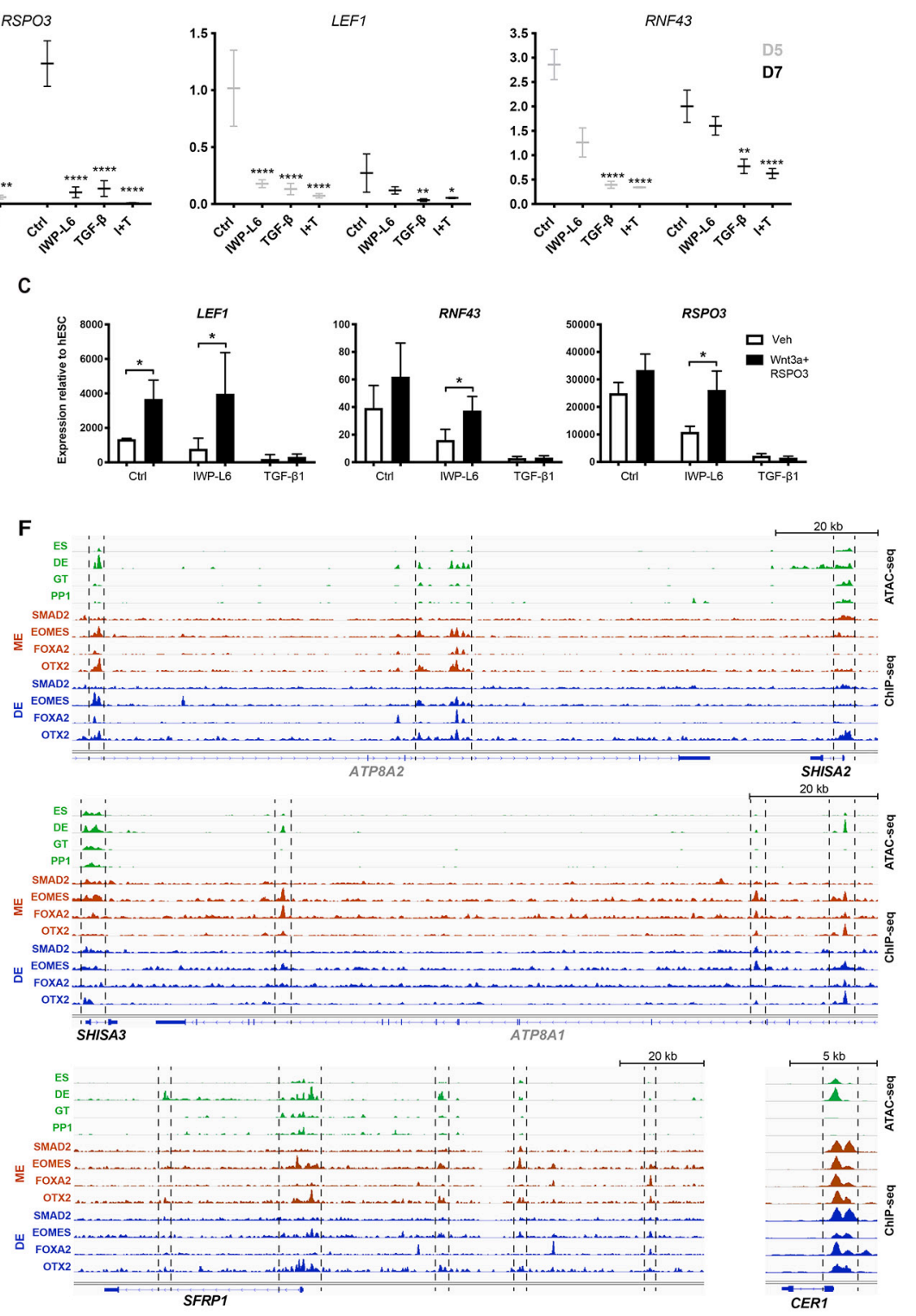

Figure 3 
bioRxiv preprint doi: https://doi.org/10.1101/2021.07.15.452587; this version posted July 16, 2021. The copyright holder for this preprint

(which was not certified by peer review) is the author/funder, who has granted bioRxiv a license to display the preprint in perpetuity. It is made available under aCC-BY-NC-ND 4.0 International license.

A

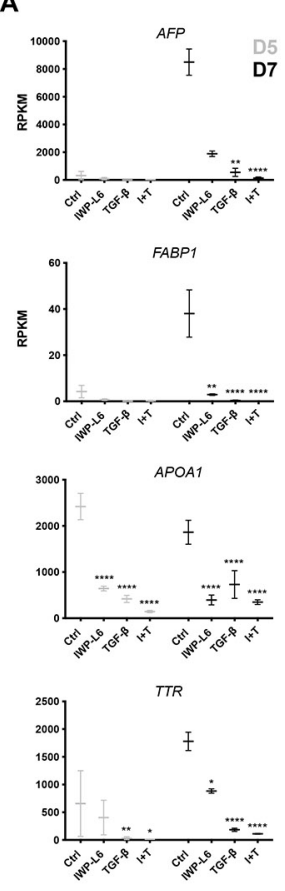

$\mathrm{F}$

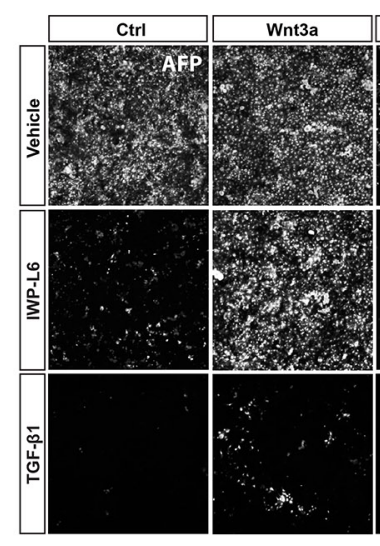

I

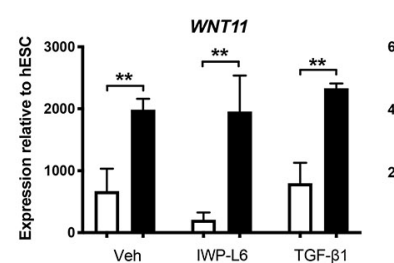

J

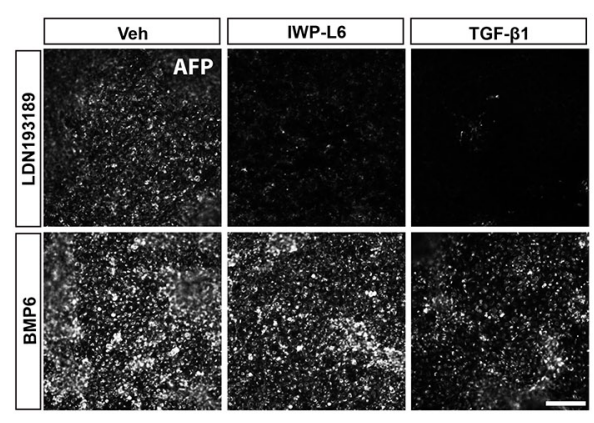

B

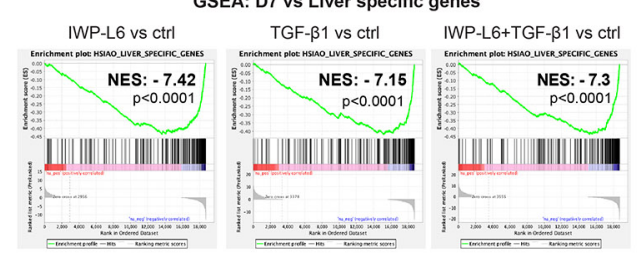

C GSEA: D7 cells vs dorsal pancreas (DP) and
hepatic cords (HC) from human CS13 embryos

hepatic cords (HC) from human
IWP-L6 vs ctrl 13 embryos

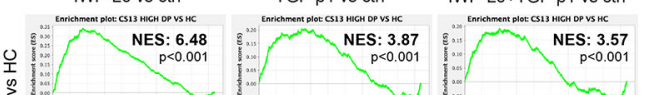

|
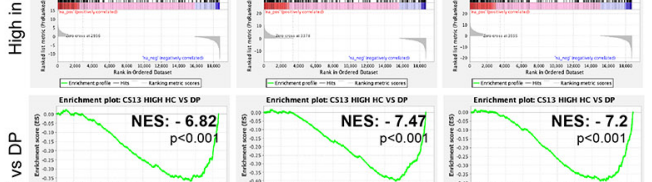

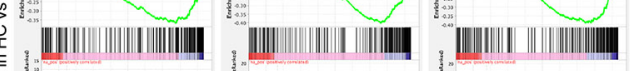

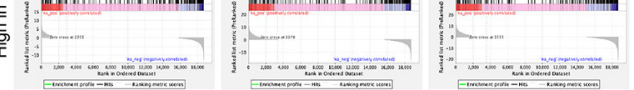

D
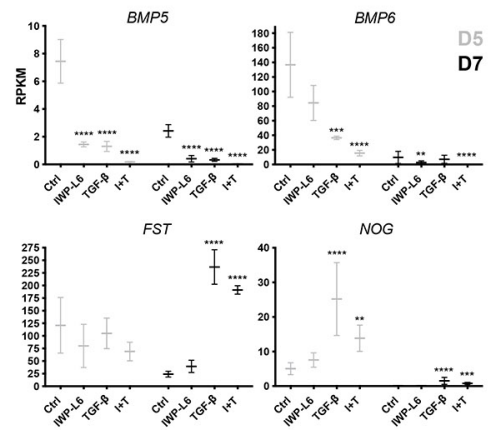

E
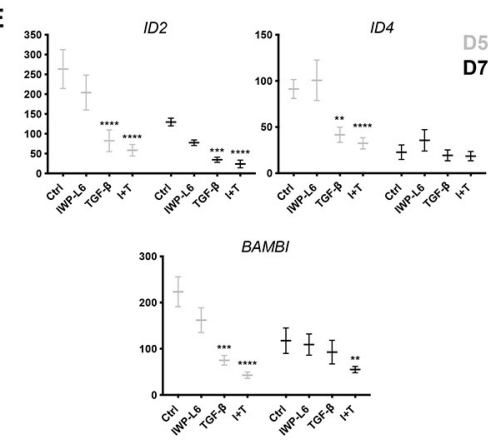

H

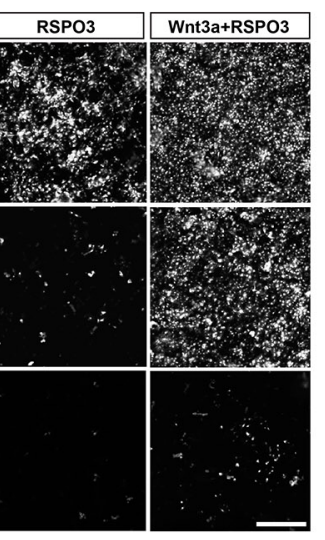

LEF1
G
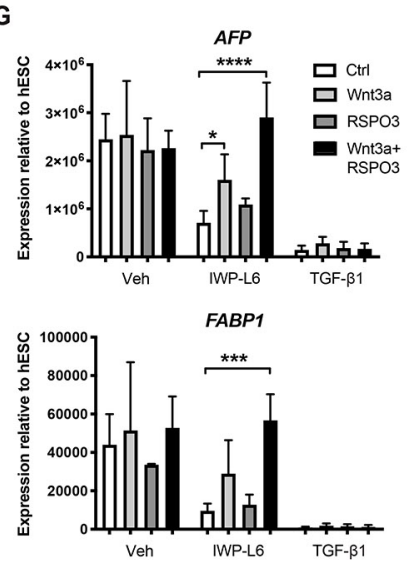

RSPO3
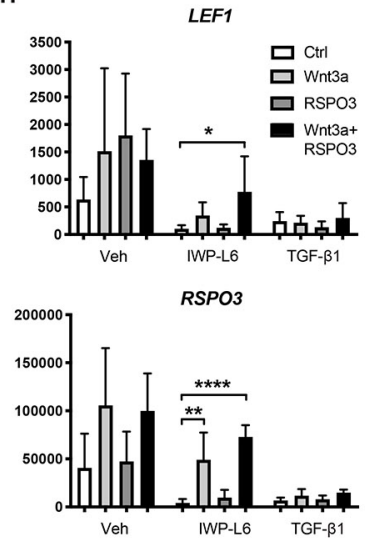

RNF43
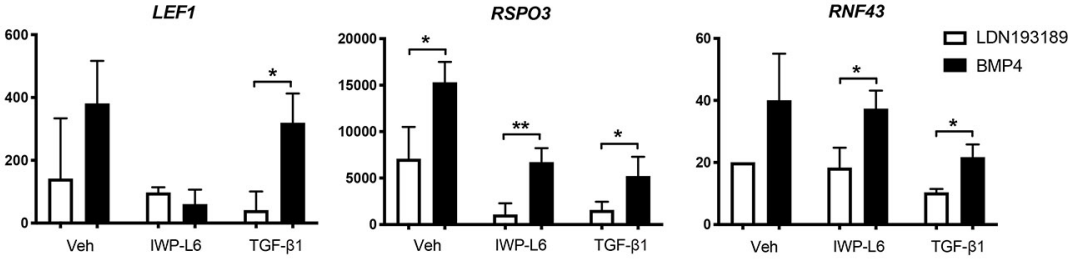

K Vinculin

L
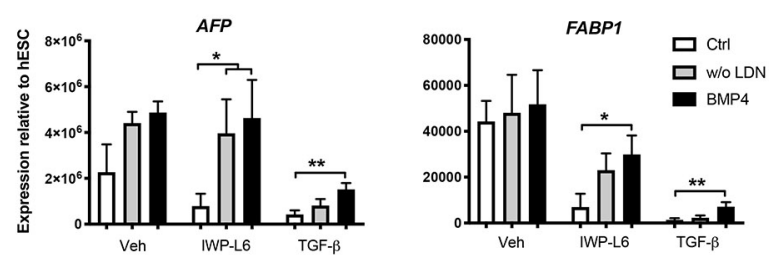

Figure 4 
bioRxiv preprint doi: https://doi.org/10.1101/2021.07.15.452587; this version posted July 16, 2021. The copyright holder for this preprint (which was not certified by peer review) is the author/funder, who has granted bioRxiv a license to display the preprint in perpetuity. It is made available under aCC-BY-NC-ND 4.0 International license.

A

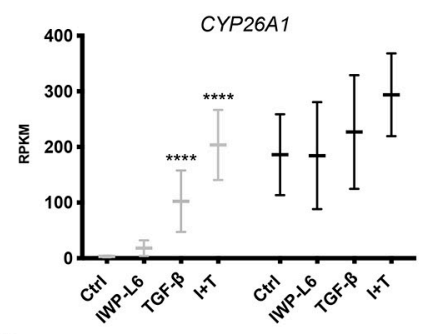

C
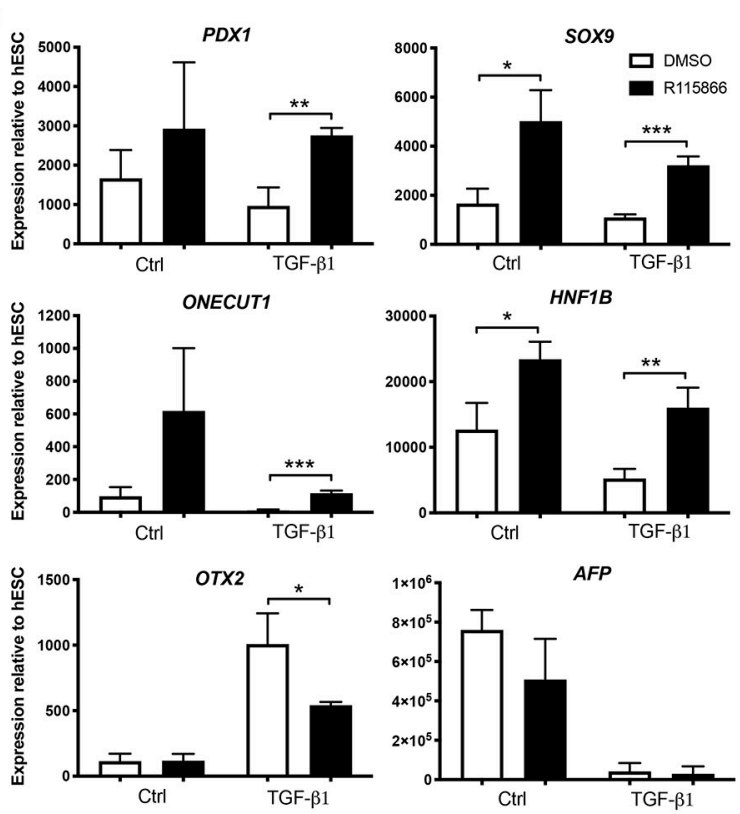

D

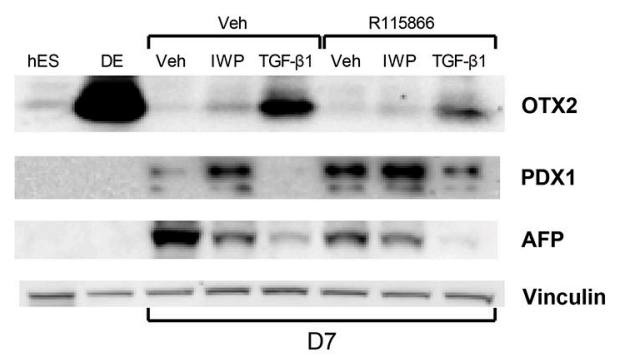

B

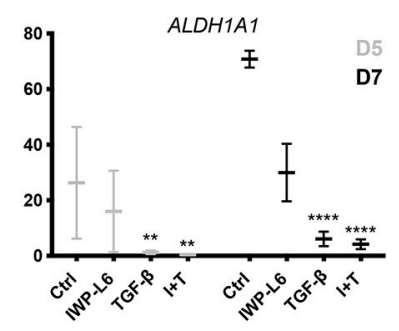

E

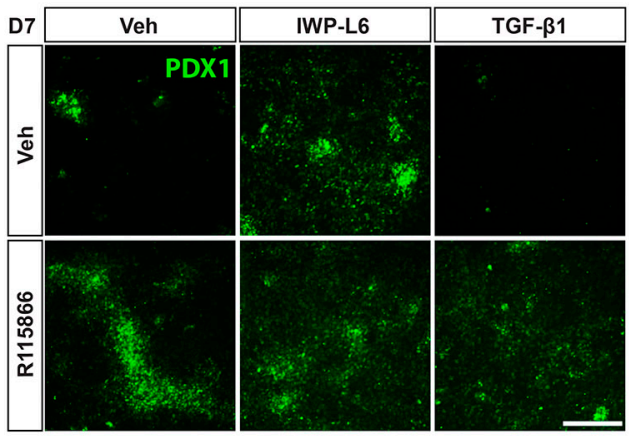

F

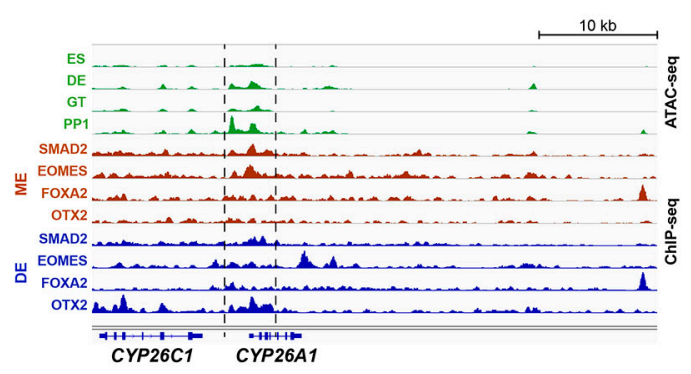

Figure 5 